\title{
Synthetic Aperture Radar Processing by a Multiple Scale Neural System for Boundary and Surface Representation
}

\author{
Stephen Grossberg ${ }^{1}$, Ennio Mingolla ${ }^{2}$, and James Williamson ${ }^{3}$ \\ Department of Cognitive and Neural Systems \\ and \\ Center for Adaptive Systems \\ Boston University \\ 111 Cummington Street, Boston, MA 02215
}

January, 1995

To appear in the 1995 Neural Networks special issue on

Automatic Target Recognition

\footnotetext{
${ }^{1}$ Supported in part by the Air Force Office of Scientific Reserach (AFOSR F49620-92-J0499), the Advanced Research Projects Agency (ONR N00014-92-J-4015), and the Office of Naval Research (ONR N00014-95-1-0409 and ONR N00014-95-1-0657).

${ }^{2}$ Supported in part by the Air Force Office of Scientific Research (AFOSR F49620-92-J0334) and the Office of Naval Research (ONR N00014-94-1-0597).

${ }^{3}$ Supported in part by the Air Force Office of Scientific Research (AFOSR F49620-92J-0225), the Advanced Research Projects Agency (ONR N00014-92-J-4015), the National Science Foundation (NSF IRI-90-24877), and the Office of Naval Research (ONR N0001491-J-4100).

${ }^{4}$ Acknowledgments: The authors wish to thank Diana Meyers for her valuable assistance in the preparation of the manuscript.
} 


\begin{abstract}
A neural network model of boundary segmentation and surface representation is developed to process images containing range data gathered by a synthetic aperture radar (SAR) sensor. The boundary and surface processing are accomplished by an improved Boundary Contour System (BCS) and Feature Contour System (FCS), respectively, that have been derived from analyses of perceptual and neurobiological data. BCS/FCS processing makes structures such as motor vehicles, roads, and buildings more salient and interpretable to human observers than they are in the original imagery. Early processing by ON cells and OFF cells embedded in shunting centersurround network models preprocessing by lateral geniculate nucleus (LGN). Such preprocessing compensates for illumination gradients, normalizes input dynamic range, and extracts local ratio contrasts. ON cell and OFF cell outputs are combined in the BCS to define oriented filters that model cortical simple cells. Pooling ON and OFF outputs at simple cells overcomes complementary processing deficiencies of each cell type along concave and convex contours, and enhances simple cell sensitivity to image edges. Oriented filter outputs are rectified and outputs sensitive to opposite contrast polarities are pooled to define complex cells. The complex cells output to stages of shortrange spatial competition (or endstopping) and orientational competition among hypercomplex cells. Hypercomplex cells activate long range cooperative bipole cells that begin to group image boundaries. Nonlinear feedback between bipole cells and hypercomplex cells segments image regions by cooperatively completing and regularizing the most favored boundaries while suppressing image noise and weaker boundary groupings. Boundary segmentation is performed by three copies of the BCS at small, medium, and large filter scales, whose subsequent interaction distances covary with the size of the filter. Filling-in of multiple surface representations occurs within the FCS at each scale via a boundary-gated diffusion process. Diffusion is activated by the normalized LGN ON and OFF outputs within ON and OFF filling-in domains. Diffusion is restricted to the regions defined by gating signals from the corresponding BCS boundary segmentation. The filled-in opponent ON and OFF signals are subtracted to form double opponent surface representations. These surface representations are shown by any of three methods to be sensitive to both image ratio contrasts and background luminance. The three scales of surface representation are then added to yield a final multiple-scale output. The BCS and FCS are shown to perform favorably in comparison to several other techniques for speckle removal.
\end{abstract}




\section{Introduction}

Synthetic aperture radar sensors can produce range imagery of high spatial resolution under difficult weather conditions (Munsen, O’Brien, and Jenkins, 1983; Munsen and Visentin, 1989) but the image data presents some difficulties for interpretation by human observers or automatic recognition systems. Among these difficulties is the large dynamic range (five orders of magnitude) of the sensor signal (see Figures 1a and 2a), which requires some type of nonlinear compression merely for an image to be represented and viewed on a typical computer monitor. Figures $1 \mathrm{~b}$ and $2 \mathrm{~b}$ (Top Right) show SAR images in which the logarithm of each pixel value is displayed to reduce the dynamic range. One major problem is image speckle, which is generated by coherent processing of radar signals, and has characteristics of random multiplicative noise.

To date, many approaches for speckle suppression have relied on simple statistical models for the signal and the noise which are insufficient for accurately representing natural scenes. Processing based on these models has thus tended to suppress the signal as well as the speckles (Lee, 1983). Other approaches have used the iterative application, within a small window, of nonlinear filtering techniques which aim to preserve the signal while smoothing speckle noise. Our approach capitalizes instead on the form-sensitive operations of a neural network model in order to detect and enhance structure based on information over large, variably sized and variably shaped regions of the image, as illustrated in Figures $1 \mathrm{~d}$ and 2d. In particular, the multi-scale implementation of the neural model reported here is capable of exploiting and combining information from several nested neighborhoods of a given image location to determine the final intensity value to be displayed for that pixel. By "neighborhood" is here meant a region whose form varies as a function of nearby image data, not some fixed (weighted) radial function for all pixel locations.

\section{Description of the Approach}

The neural network model used here is a refinement of the Boundary Contour System (BCS) for boundary segmentation that was introduced by Grossberg and Mingolla (1985a, 1985b, 1987) and the Feature Contour System (FCS) for surface representation that was introduced by Cohen and Grossberg (1984) and Grossberg and Todorović (1988) through an analysis of biological vision. Several of these improvements were introduced in Cruthirds, et al. (1992). Taken together, the BCS and FCS form part of the FACADE theory of biological and machine vision (Grossberg, 1994), so called because the acronym FACADE stands for the representations of Form-And-Color-And- 

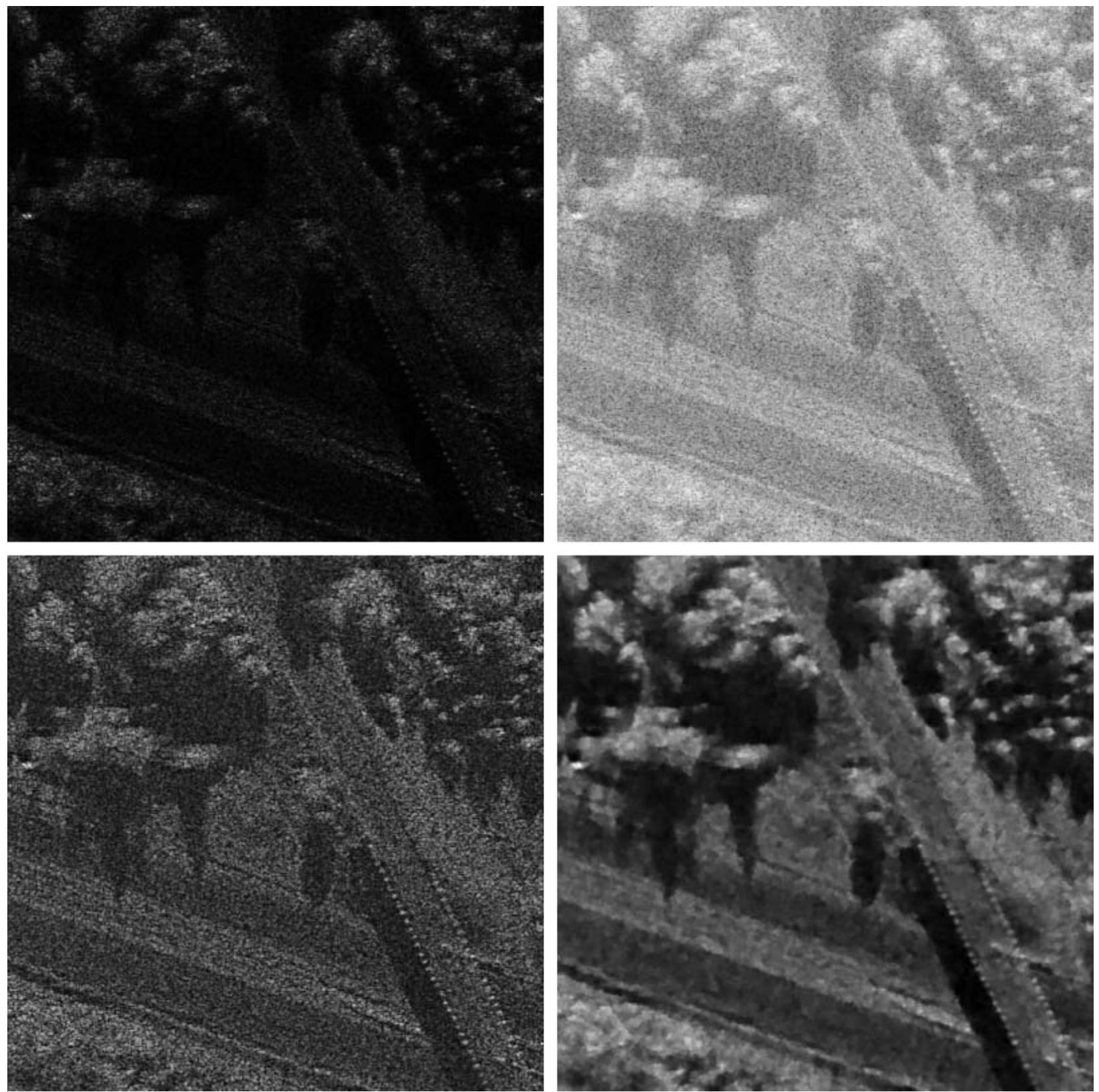

Figure 1: (a) Top Left: Unprocessed SAR image of upstate New York scene consisting of highway with bridge overpass. (b) Top Right: Logarithm-transformed SAR image. (c) Bottom Left: Stage 1 result averaged across spatial scales. (d) Bottom Right: Multiple scale BCS/FCS result from processing Model II on original SAR image. 

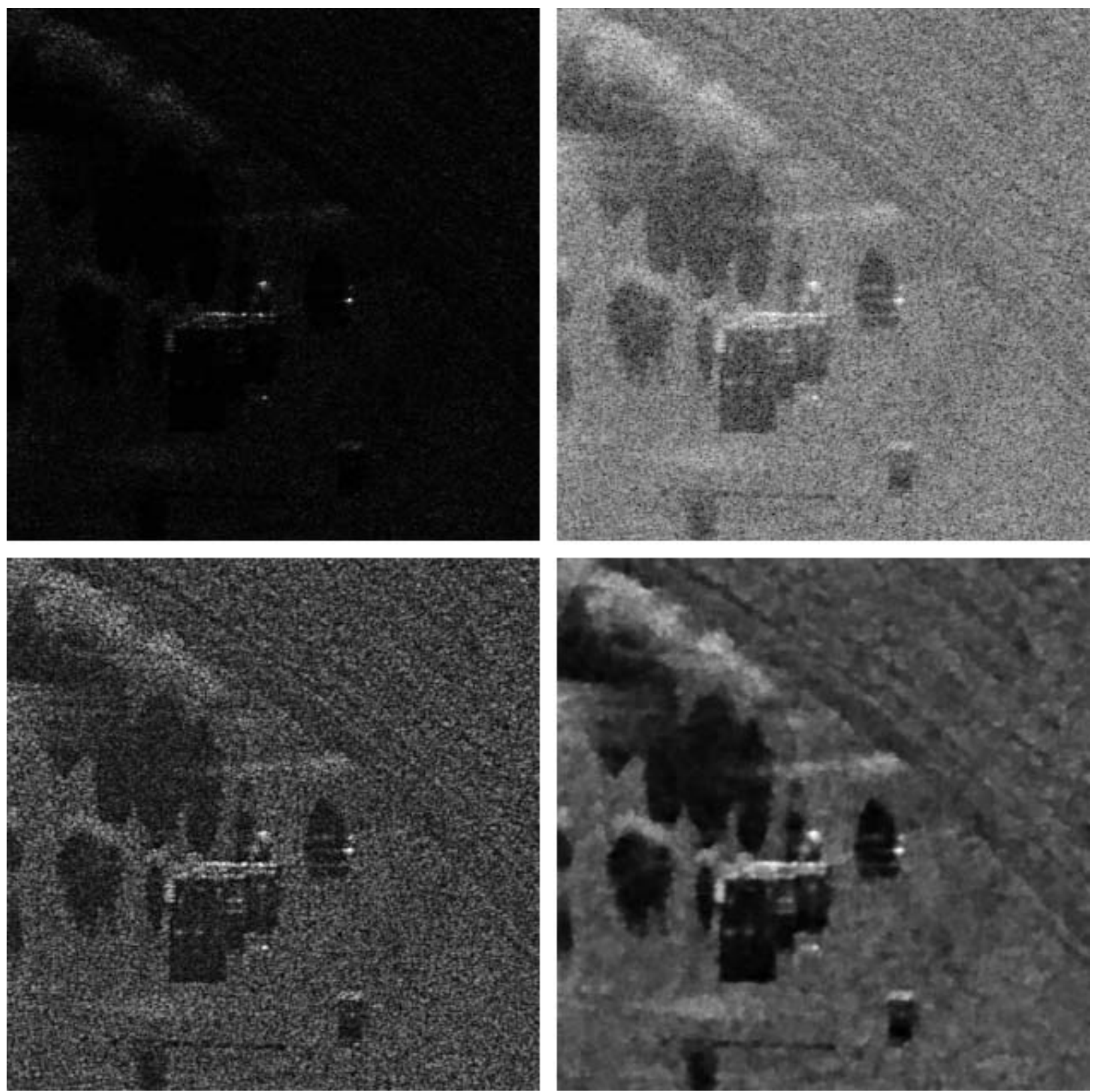

Figure 2: (a) Top Left: Unprocessed SAR image of upstate New York scene consisting of house, trees, and road. (b) Top Right: Logarithm-transformed SAR image. (c) Bottom Left: Stage 1 result averaged across spatial scales. (d) Bottom Right: Multiple scale BCS/FCS result from processing Model II on original SAR image. 
DEpth that are suggested to occur at the final FCS surface representations of the full binocular theory.

In the present work, only monocular, or single detector, processing is described, so the model is considerably simpler than its binocular version. As summarized in Figure 3, ON cells and OFF cells preprocess the inputs as parts of on-center off-surround and off-center on-surround networks, respectively. Preprocessing compensates for illumination gradients, normalizes input dynamic range, and extracts local ratio contrasts. ON and OFF cell outputs are processed in parallel by both the BCS and the FCS.

The BCS combines the ON and OFF cell outputs to detect, regularize, and complete coherent boundary representations, while suppressing image noise, using multiple-scale filtering and cooperative-competitive feedback interactions. Multiple copies of the BCS are defined, each corresponding to a different receptive field size. Each BCS copy inputs to a corresponding copy of the FCS at which filling-in of a surface representation occurs. Filling-in is initiated by normalized input patterns from the ON and OFF cell preprocessor. Target ON and OFF cells of the FCS are activated and diffuse activity to their nearest neighbors. Topographic signals from the BCS boundaries define barriers to the diffusion process.

These filling-in processes are based on the ON and OFF signals that survive after preprocessing compensates for illumination gradients. The filled-in surface representations hereby generate perceptual constancies, such as brightness constancy, under variable illumination conditions (Grossberg and Todorović, 1988). Filled-in ON and OFF surface representations are then combined by any of three methods to define surface representations that combine both ratio contrast and image luminance information. These surface representations at different scales are then topographically averaged to generate the output surface representation. Figure 3 shows the processing stages of the BCS/FCS at a single scale for each model. Figure 4 shows how the scales are combined subsequent to the FCS filling-in stage.

In Section 3, we summarize illustrative SAR image processing results. Section 4 provides an intuitive summary of the BCS and FCS processing stages. Section 5 describes the model equations, along with interpretive remarks. Section 6 compares BCS/FCS processing with alternative methods for processing SAR images. 

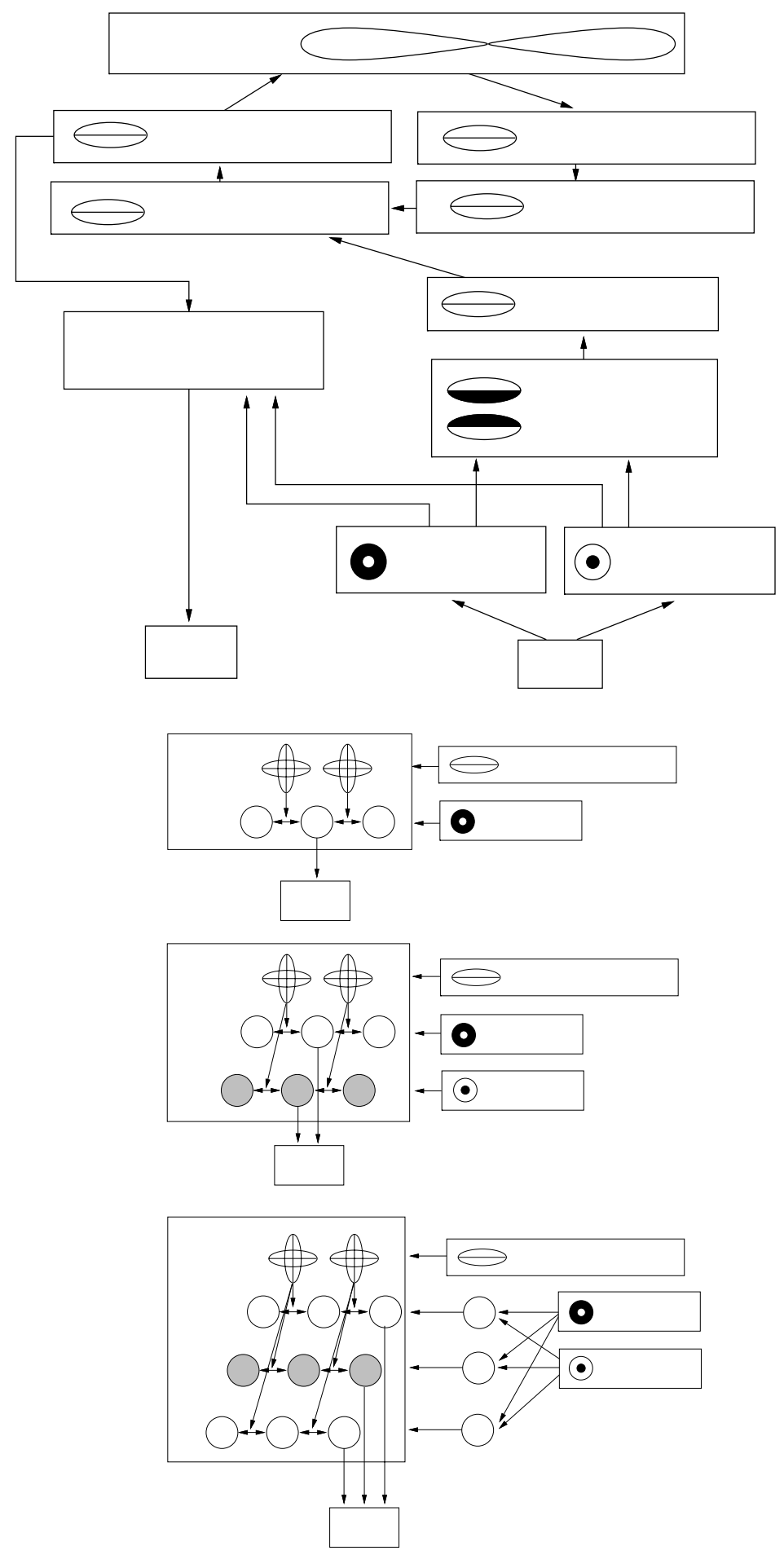

Figure 3: (a) A single scale of processing in the BCS/FCS model. Diffusive surface filling-in by (b) Model I, (c) Model II, and (d) Model III. 


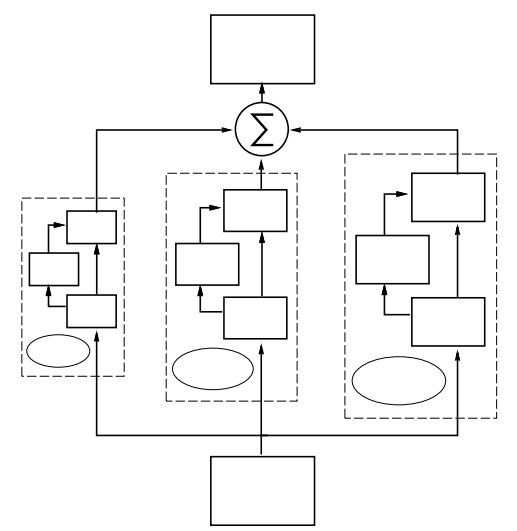

Figure 4: A multiple-scale BCS/FCS model.

\section{Methods and Results}

The SAR images were obtained using a 35-GHz synthetic aperture radar with $1 \mathrm{ft}$ by $1 \mathrm{ft}$ resolution and a slant range of $7 \mathrm{~km}$ (Novak, Burl, Chaney, and Owirka, 1990). Figure 1 shows a SAR image and the result of the multiple-scale BCS/FCS model II applied to the image. Figure 1a shows the original SAR image, Figure $1 \mathrm{~b}$ the logarithmically transformed $\left(\log _{10}\right)$ version of the original image for comparison, Figure $1 \mathrm{c}$ the Stage 1 center-surround processing result of the original image averaged across spatial scales, and Figure 1d the multiple-scale output of the BCS/FCS system. The image is from upstate New York, of a highway with bridge overpass. The original 512x512 pixel image was reduced via gray-level consolidation to 400x400 pixels before processing. Specifically, when the number of pixels is reduced, each new pixel (if envisioned overlayed on the original 2D grid) is larger than the original pixels. Thus the value of a new pixel is an average of the old pixels that it overlays, with the contribution of each of the old pixels proportional to how much of it is overlayed. Figure 2 shows analogous results for an image consisting of a house with some surrounding trees and a small road. This image was reduced from 400x400 to 312x312 pixels before processing.

Figures 1 and 2 illustrate the main results obtained by the multiple-scale BCS/FCS system. First, Stage 1 center-surround processing compresses dynamic range, performs a partial local image normalization, and contrast-enhances local image intensities to yield a viewable image, resulting in 
the transform from Figures $1 \mathrm{a}$ and $2 \mathrm{a}$ to Figures $1 \mathrm{c}$ and $2 \mathrm{c}$, respectively. Unlike the logarithmic transformation, which also compresses dynamic range, yielding Figures $1 \mathrm{~b}$ and 2b, Stage 1 output from ON and OFF cells in Figure 3 contrast-enhances local structures at each spatial scale. Stage 1 output is next processed with rectified oriented contrast-sensitive filters at Stages 2 and 3 of Figure 3. At Stages 4 to 8, cooperative-competitive feedback at different spatial scales enhances and completes colinear or nearly colinear structures and thereby segments the image into regions. Stage 1 output then diffuses within these region boundaries, but not between them, at Stage 9, thus smoothing over image speckle while preserving meaningful intensity differences between regions. After these filled-in surface representations are obtained, they are combined as outlined above to yield the final output of Figures $1 d$ and $2 d$.

\section{Intuitive Description of BCS and FCS}

Grossberg (1984) and Cohen and Grossberg (1984) introduced the BCS and FCS models. Grossberg and Mingolla $(1985 \mathrm{a}, 1985 \mathrm{~b}, 1987)$ developed the BCS model to simulate how the visual system detects, completes, and regularizes boundary segmentations in response to a variety of retinal images. Such segmentations can be defined by regions of different luminance, color, texture, shading, or stereo signals. The BCS computations for single-scale monocular processing consist of a series of filtering, competitive, and cooperative stages as schematized in Figure 3 and reviewed in several reports (e.g., Grossberg, 1987a, 1994; Grossberg, Mingolla, and Todorović, 1989). The first stage, schematized as unoriented annuli in Figure 3, models in perhaps the simplest possible way the shunting on-center off-surround, and off-center on-surround, interactions at the retinal and LGN levels. These ON and OFF cells compensate for variable illumination and compute the ratio contrasts in the image.

The model LGN cells generate half-wave rectified outputs. These outputs are received by pairs of like-oriented simple cells (Stage 2 in Figure 3 ) that are sensitive to opposite contrast polarity, or direction-of-contrast. The simple cell pairs, in turn, pool their rectified and oppositely polarized output signals at like-oriented complex cells (Stage 3 ). Complex cells are hereby rendered insensitive to direction-of-contrast, as are all subsequent cell types in the model. Complex cells activate hypercomplex cells through an on-center off-surround network (Stage 4) whose off-surround carries out an endstopping operation. In this way, complex cells excite hypercomplex cells of the same orientation and position, while inhibiting hypercomplex cells of the same orientation at nearby 
positions. One role of this spatial competition is to spatially sharpen the neural responses to oriented luminance edges. Another role is to initiate the process at Stage 5 called end cutting, whereby boundaries are formed that abut a line end at orientations perpendicular or oblique to the orientation of the line itself (Grossberg, 1987a; Grossberg and Mingolla, 1985b).

Output from the higher-order hypercomplex cells feed into cooperative bipole cells at Stage 6. The bipole cells initiate long-range boundary grouping and completion. Bipole cells realize a type of statistical AND gate, since they fire if both of their receptive fields are sufficiently activated by appropriately oriented hypercomplex cell inputs. Bipole cells hereby realize a type of long-range cooperation among the outputs of active hypercomplex cells. For example, a horizontal bipole cell, as in Figure 3, is excited by activation of horizontal hypercomplex cells that input to its horizontally oriented receptive fields. A horizontal bipole cell is also inhibited by activation of vertical hypercomplex cells. This inhibition prevents boundaries from being colinearly completed across regions that contain sufficiently many perpendicular or oblique contrasts, a property called spatial impenetrability (Grossberg, 1987a; Grossberg and Mingolla, 1985b).

Bipole cells were predicted to exist in Cohen and Grossberg (1984) and Grossberg (1984) shortly before cortical cells in area V2 with similar properties were reported by von der Heydt, Peterhans, and Baumgartner (1984). At around the time of the von der Heydt et al. report, Grossberg and Mingolla (1985a, 1985b) used bipole cell properties to simulate and explain data about illusory contour formation, neon color spreading, and texture segregation, among other topics. These same properties play a role in our simulations of SAR data.

Bipole cells generate feedback signals to like-oriented hypercomplex cells within Stages 7 and 8 of Figure 3. These feedback signals help to create and enhance spatially and orientationally consistent boundary groupings, while inhibiting inconsistent ones. In particular, bipole cell outputs compete across orientation at each position within Stage 7 to select the cooperatively most favored orientation, or orientations. These outputs then undergo spatial competition that excites cells at the same orientation and position while inhibiting cells at nearby positions. Cells which derive the most cooperative support from bipole grouping after these competitive selection processes thereupon further excite the corresponding bipole cells. This cycle of bottom-up and top-down interaction between hypercomplex cells and bipole cells rapidly converges to a final boundary segmentation. Feedback among bipole cells and hypercomplex cells hereby drives a resonant cooperative-competitive decision process that completes the statistically most favored boundaries, suppresses less favored boundaries, and coherently binds together appropriate feature combinations in the image. 
Cohen and Grossberg (1984) and Grossberg and Todorović (1988) developed the FCS model to simulate many data about brightness perception. Arrington (1994) has shown that the GrossbergTodorović (1988) model also accurately simulates the dynamics of brightness filling-in as reported in the psychophysical experiments of Paradiso and Nakayama (1991). The BCS produces boundary signals that act as barriers to diffusion within the FCS in response to ON and OFF inputs from which the illuminant has been discounted. As diagrammed in Figure 3, these boundary signals act to gate diffusion of signals from the ON and OFF cells at Stage 9. That is, for image pixels through which no boundary signals pass, resulting intensity values become more homogeneous as the diffusion evolves. Where boundary signals intervene, however, they block the diffusion, leaving a resulting difference of intensity level on either side of the boundary signal. The result of such boundary-gated diffusion is a form-sensitive computation that adapts to each unique combination of image inputs, rather than a correlation derived through a fixed kernel.

The ON and OFF signals may or may not be combined to generate the final FCS surface representation. There are several related ways to do this that all lead to essentially equivalent results. The basic idea in all cases is to combine FCS surface measures that depend upon both the ratio contrasts and the averaged background luminances of the image. The simplest variation is the model I whose output is summarized in Figure 5. Here the ON responses themselves are used to fill-in surface properties (Cohen and Grossberg, 1984; Grossberg and Todorović, 1988). That is why model I is called the ON cell model. All the models including model I heavily use the fact that the ON cells are the result of shunting on-center off-surround, or cooperative-competitive, processing that computes a measure of local ratio contrast. In addition, the excitatory and inhibitory parameters of the cells are chosen asymmetrically in model I, so that the ratio contrasts add to a constant background activity level which is modulated by a locally averaged luminance level in response to dense imagery.

In order to compute a background activity level that covaries more generally with locally averaged image luminances, both ON and OFF cell responses are used. Here, the OFF responses are subtracted from the ON responses, either before or after the filling-in stage. This strategy was introduced in Grossberg (1987b) and has been applied in several studies since; e.g., Grossberg (1994), Grossberg and Wyse (1991), Neumann (1993), and Pessoa, Mingolla, and Neumann (1995). Such a subtraction of OFF (off-center on-surround) signals from ON (on-center off-surround) signal cells is said to create double opponent cells, since it combines two successive competitive (or opponent) interactions. 


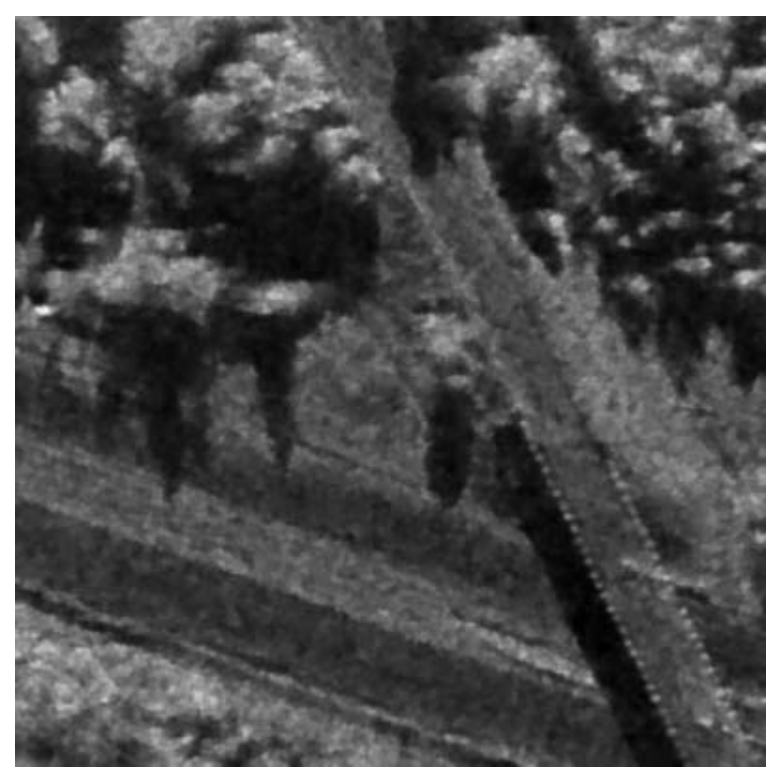

Figure 5: Processing by Model I scales of the bridge-overpass image. Compare with Model II result in Figure 1d.

In perhaps the simplest double opponent computation, that of Grossberg and Wyse (1991), the OFF cells have a higher tonic, or baseline, activity than do the ON cells. When the OFF cell responses are subtracted from the $\mathrm{ON}$ cell responses, there are two terms: one is sensitive to ratio contrast and the other, which arises from the asymmetric baseline activities, increases as a function of a low-pass nonlinearly-compressed luminance estimate. The net double opponent signal diffuses across an FCS filling-in domain, or FIDO. Alternatively, the ON and OFF inputs could first diffuse within their own ON and OFF FIDOs, each gated by the same boundary segmentation, before the net ON-minus-OFF double-opponent response is computed. Let us call this the asymmetric ON/OFF model, or model II. Figure 1 shows the output of model II to the same image that is processed by model I in Figure 5.

A related approach, that of Neumann (1993), subtracts OFF cell outputs from ON cell outputs, where both cell types have the same, possibly zero, tonic activity. This double opponent operation generates a measure of relative contrast only. The rectified output signal from this operation is allowed to fill-in within a FIDO. Likewise, rectified OFF-minus-ON signals fill-in their own FIDO. In addition, ON plus OFF activities are added, without filling-in, to the filled-in ON-minus-OFF activities to provide a baseline that is sensitive to background luminance, and the filled-in OFFminus- $\mathrm{ON}$ activities are divided from them. These difference (ON-OFF) and sum (ON+OFF) operations are reminiscent of the L-M color computation and L+M luminance computation that 


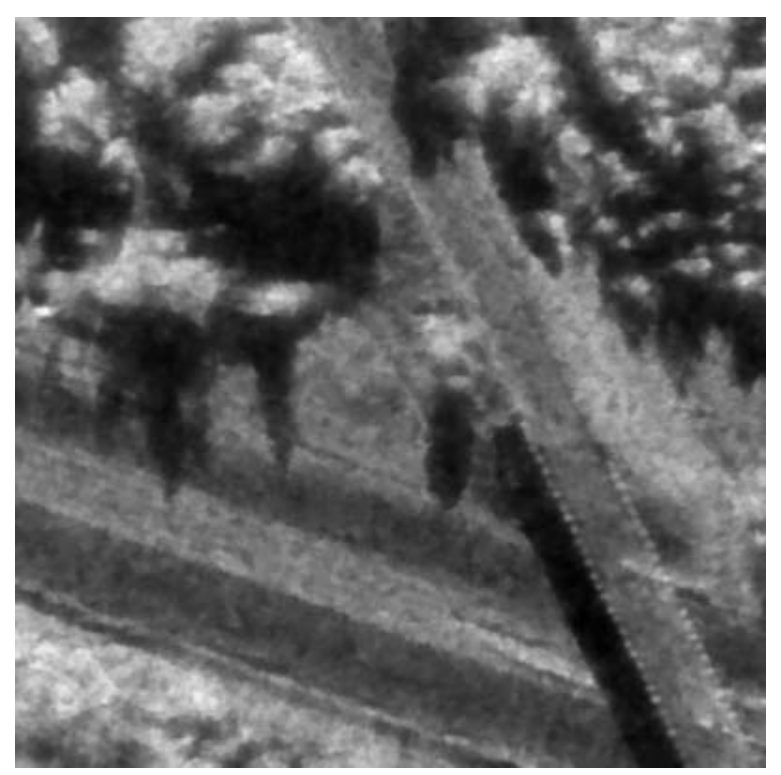

Figure 6: Same as Figure 5 for Model III.

takes place between long (L) and medium (M) wavelength retinal cone channels (Mollon and Sharpe, $1983)$.

For SAR images, combining the ON+OFF response, without filling-in, to the filled-in $\mathrm{ON}-\mathrm{OFF}$ and $\mathrm{OFF}-\mathrm{ON}$ responses reintroduces image speckle and other distortions that filling-in helps to overcome. We therefore modify this method by filling-in both the difference and sum responses before combining them. Let us call this revised model the symmetric ON/OFF model, or model III. Figure 6 shows the output of Model III to the same image as in Figure 1. Comparison of Figures 1, 5 , and 6 shows that all three model variants produce similar outputs in response to the SAR data.

These BCS/FCS computations are computed at three different scales in order to enhance image structures of different sizes. As diagrammed in Figure 4, the filled-in surface representations of the different scales are added to yield a final multiple scale output.

\section{Mathematical Description of BCS and FCS}

\subsection{Stage 1: Shunting ON and OFF Center-Surround Networks}

The first processing stage performs a partial local normalization of image intensities. This is accomplished by two shunting center-surround systems. The first, an on-center off-surround network, corresponds to an ON channel of the visual pathway. The second shunting network, with an offcenter and on-surround, corresponds to an OFF channel. In each case the equilibrium activities of 
the networks contains both a DOG (Difference of Gaussians) term in the numerator, which detects contrast differences, and a SOG (Sum of Gaussians) term in the denominator which compensates for the level of illumination, thereby discounting the illuminant. The two networks differ in the sign of their responses to a given light-to-dark (left-to-right) step transition, as the ON channel responds positively on the left side of the step, and the OFF channel responds positively on the right side of the step (negative outputs are set to zero). The outputs of the ON and OFF cells, beside feeding into Stage 2, are also employed as the FCS signals that feed into Stage 9. The unprocessed SAR image (not logarithmically transformed) is input into the equilibrium forms of the following shunting on-center off-surround, and off-center on-surround, differential equations that define the activities of ON and OFF preprocessing cells (Grossberg, 1973).

\section{ON Opponent Cell Activation}

$$
\frac{d}{d t} x_{i j}^{g}=-D\left(x_{i j}^{g}-E\right)+\sum_{(p, q)}\left[\left(U-x_{i j}^{g}\right) C_{i j p q}^{g}-\left(x_{i j}^{g}+L\right) S_{i j p q}^{g}\right] I_{p q} .
$$

\section{OFF Opponent Cell Activation}

$$
\frac{d}{d t} \bar{x}_{i j}^{g}=-D\left(\bar{x}_{i j}^{g}-\bar{E}\right)+\sum_{(p, q)}\left[\left(U-\bar{x}_{i j}^{g}\right) S_{i j p q}^{g}-\left(\bar{x}_{i j}^{g}+L\right) C_{i j p q}^{g}\right] I_{p q} .
$$

In (1) and (2), the center and surround kernels are

$$
\begin{aligned}
& C_{i j p q}^{g}=\frac{C}{2 \pi \sigma_{c g}^{2}} \exp \left\{-\frac{(i-p)^{2}+(j-q)^{2}}{2 \sigma_{c g}^{2}}\right\}, \\
& S_{i j p q}^{g}=\frac{S}{2 \pi \sigma_{s g}^{2}} \exp \left\{-\frac{(i-p)^{2}+(j-q)^{2}}{2 \sigma_{s g}^{2}}\right\} .
\end{aligned}
$$

The ON channel activity at position $(i, j)$ and scale $g$ is denoted by $x_{i j}^{g}$ in (1), and the corresponding OFF channel activity is denoted by $\bar{x}_{i j}^{g}$ in $(2)$. Term $I_{p q}$ is the input to position $(p, q)$ of both channels. Note that the center kernel (C) and the surround kernel (S) of the ON cells in (1) are reversed in (2) to become the surround and center kernels, respectively, of the OFF cells. The cell activities are evaluated at equilibrium and rectified, yielding the ON and OFF output signals:

\section{ON Opponent Output Signal}

$$
X_{i j}^{g}=\left[\frac{D E+\sum_{(p, q)}\left(U C_{i j p q}^{g}-L S_{i j p q}^{g}\right) I_{p q}}{D+\sum_{(p, q)}\left(C_{i j p q}^{g}+S_{i j p q}^{g}\right) I_{p q}}\right]^{+}
$$




\section{OFF Opponent Output Signal}

$$
\bar{X}_{i j}^{g}=\left[\frac{D \bar{E}+\sum_{(p, q)}\left(U S_{i j p q}^{g}-L C_{i j p q}^{g}\right) I_{p q}}{D+\sum_{(p, q)}\left(C_{i j p q}^{g}+S_{i j p q}^{g}\right) I_{p q}}\right]^{+} .
$$

where

$$
[\omega]^{+}=\max (\omega, 0)
$$

The ON and OFF channels have a DC level that is determined by a baseline activity level $E$ or $\bar{E}$, respectively (Grossberg and Wyse, 1991).

Equations (5) and (6), respectively, compute on-center off-surround, and off-center on-surround, normalized ratio contrasts of the input image. The equations are applied at three spatial scales, $\sigma_{c g}$ and $\sigma_{s g}, g=0,1,2$, which are defined by the standard deviations of the center and surround Gaussian kernels in (3) and (4). See Table 1. The center kernels are small and constant across scales to yield high spatial frequency detail at all scales, while the surround kernels increase with scale in order to modulate the center response with lower spatial frequency information at larger scales. Other parameters are for models I-III are found in Tables 1-3. For example, large SAR image values, having ranges of approximately [49-14,281] and [53-25,254] in Figures 1a and 2a, respectively, necessitate the large "decay" parameter value of $D=2000$ in (5) and (6) in order to prevent information about a local image intensity from being completely normalized.

With $E$ and $\bar{E}$ chosen so that equilibrium $\mathrm{ON}$ and $\mathrm{OFF}$ activities are positive, subtraction of the OFF channel output from the ON channel output yields

\section{ON Double Opponent Cell Activation}

$$
d_{i j}^{g}=X_{i j}^{g}-\bar{X}_{i j}^{g}=\frac{(U+L) \sum_{(p, q)}\left(C_{i j p q}^{g}-S_{i j p q}^{g}\right) I_{p q}}{D+\sum_{(p, q)}\left(C_{i j p q}^{g}+S_{i j p q}^{g}\right) I_{p q}}-\frac{D(\bar{E}-E)}{D+\sum_{(p, q)}\left(C_{i j p q}^{g}+S_{i j p q}^{g}\right) I_{p q}}
$$

Likewise, the OFF double opponent cell activation is defined by

$$
\bar{d}_{i j}^{g}=\bar{X}_{i j}^{g}-X_{i j}^{g}
$$

Equation (8), and likewise equation (9), shows that the effects of subtracting OFF from ON activities result in an activation profile whose first term is sensitive to the ratio-contrasts in the 
Table 1: Parameters for Model II

\begin{tabular}{|c|c|c|c|}
\hline Name & Description & Value & Equation(s) \\
\hline \multicolumn{4}{|c|}{ Stage 1: Shunting ON and OFF Center-Surround Networks } \\
\hline$\sigma_{c g}, g=0,1,2$ & Center blurring space constants & 0.3 & $(3)$ \\
\hline$\sigma_{s 0}$ & Surround blurring space constant & 1.2 & (4) \\
\hline$\sigma_{s 1}$ & Surround blurring space constant & 3.6 & (4) \\
\hline$\sigma_{s 2}$ & Surround blurring space constant & 10.8 & (4) \\
\hline$C, S$ & Center and Surround coefficients & 1.0 & $(3),(4)$ \\
\hline$U$ & Polarization constant & 1.0 & $(1),(2)$ \\
\hline$L$ & Hyperpolarization constant & 1.0 & $(1),(2)$ \\
\hline$D$ & Activation decay & 2000.0 & $(1),(2)$ \\
\hline$E$ & ON baseline activity level & 0.5 & (1) \\
\hline $\bar{E}$ & OFF baseline activity level & 1.0 & (1) \\
\hline \multicolumn{4}{|c|}{ Stages 2 and 3: Simple and Complex Cells } \\
\hline$\sigma_{v 0}$ & Blurring space constant & 0.75 & (19) \\
\hline$\sigma_{v 1}$ & Blurring space constant & 1.5 & $(19)$ \\
\hline$\sigma_{v 2}$ & Blurring space constant & 3.0 & (19) \\
\hline$\sigma_{h g}, g=0,1,2$ & Blurring space constants & $3 \sigma_{v g}$ & $(19)$ \\
\hline$A$ & Scaling factor & 500.0 & $(23)$ \\
\hline$B$ & Complex cell threshold & 0.01 & $(23)$ \\
\hline \multicolumn{4}{|c|}{ Stage 4: Hypercomplex Cells, Competition I } \\
\hline$\sigma_{c g}, g=0,1,2$ & Center blurring space constants & 0.1 & $(22),(24)$ \\
\hline$\sigma_{s 0}$ & Surround blurring space constant & 1.0 & $(22),(24)$ \\
\hline$\sigma_{s 1}$ & Surround blurring space constant & 2.0 & $(22),(24)$ \\
\hline$\sigma_{s 2}$ & Surround blurring space constant & 4.0 & $(22),(24)$ \\
\hline$D$ & Activation decay & 1000.0 & $(22),(24)$ \\
\hline$U$ & Polarization constant & 1.0 & $(22),(24)$ \\
\hline$L$ & Hyperpolarization constant & 1.0 & $(22),(24)$ \\
\hline$T$ & Tonic input & 10.0 & $(22),(24)$ \\
\hline$E_{0}$ & Feedback scaling factor & 400.0 & $(23)$ \\
\hline$E_{1}$ & Feedback scaling factor & 450.0 & $(23)$ \\
\hline$E_{2}$ & Feedback scaling factor & 600.0 & $(23)$ \\
\hline \multicolumn{4}{|c|}{ Stage 5: Hypercomplex Cells, Competition II } \\
\hline$\sigma_{c}, g=0,1,2$ & Center blurring orientation constant & 0.7 & $(26)$ \\
\hline$\sigma_{s}$ & Surround blurring orientation constant & 6.0 & $(27)$ \\
\hline$C$ & Center coefficient & 0.7 & $(25),(28)$ \\
\hline$S$ & Surround coefficient & 30.0 & $(25),(28)$ \\
\hline$U$ & Polarization constant & 3.2 & $(25),(28)$ \\
\hline$L$ & Hyperpolarization constant & 0.8 & $(25),(28)$ \\
\hline$D$ & Activation decay & 1.0 & $(25),(28)$ \\
\hline
\end{tabular}


Table 1: Parameters for Model II (Cont'd)

\begin{tabular}{llcc}
\hline Name & Description & Value & Equation(s) \\
\hline Stage 6: & Bipole Cells, Long-Range Cooperation & \\
$C_{0}$ & Bipole filter size & 15 & $(32),(33)$ \\
$C_{1}$ & Bipole filter size & 29 & $(32),(33)$ \\
$C_{2}$ & Bipole filter size & 57 & $(32),(33)$ \\
$\alpha$ & Bipole lobe divisive constant & 0.015 & $(31)$ \\
$\beta$ & Distance fall-off coefficient & 0.8 & $(34)$ \\
$\mu$ & Curvature fall-off coefficient & 11.0 & $(34)$ \\
$\gamma$ & Orientational selectivity & 31.0 & $(34)$ \\
$A_{0}$ & Output threshold & 0.16 & $(35)$ \\
$A_{1}$ & Output threshold & 0.12 & $(35)$ \\
$A_{2}$ & Output threshold & 0.08 & $(35)$ \\
Stage $7:$ Hypercomplex/Bipole Cells, Feedback Competition II \\
Stage 8: Hypercomplex/Bipole Cells, Feedback Competition I \\
$\sigma_{v 0}, \sigma_{h 0}$ Blurring space constants & 0.75 & $(38)$ \\
$\sigma_{v 1}, \sigma_{h 1}$ & Blurring space constants & 1.0 & $(38)$ \\
$\sigma_{v 2}, \sigma_{h 2}$ & Blurring space constants & 2.0 & $(38)$ \\
Stage 9: Filling-in & & \\
$D$ & Activation decay & 0.05 & $(39)-(42)$ \\
$\delta$ & Permeability numerator factor & 10.0 & $(39)-(42)$ \\
$\epsilon$ & Permeability denominator factor & 1000.0 & $(39)-(42)$
\end{tabular}

Table 2: Parameters Unique to Model I

\begin{tabular}{lccc}
\hline Name & Description & Value & Equation(s) \\
\hline \multicolumn{5}{l}{ Stage 1: Shunting ON and OFF Center-Surround } & Networks \\
$L$ & Hyperpolarization constant & 0.1 & $(1),(2)$ \\
$E$ & ON baseline activity level & 0.0 & $(1)$ \\
$\bar{E}$ & OFF baseline activity level & 0.0 & $(1)$ \\
Stages 2 and 3: Simple and Complex Cells & & \\
A & Scaling factor & 1000.0 & $(23)$ \\
$B$ & Complex cell threshold & 0.0 & $(23)$ \\
Stage $6:$ Bipole Cells, Long-Range Cooperation & \\
$A_{0}$ & Output threshold & 0.08 & $(35)$ \\
$A_{1}$ & Output threshold & 0.07 & $(35)$ \\
$A_{2}$ & Output threshold & 0.05 & $(35)$ \\
Stage 9: Filling-in & & \\
$\epsilon$ & Permeability denominator factor & 2000.0 & $(39)-(42)$
\end{tabular}


Table 3: Parameters Unique to Model III

\begin{tabular}{lccc}
\hline Name & Description & Value & Equation(s) \\
\hline Stage 1: Shunting ON and OFF Center-Surround & Networks & \\
$L$ & Hyperpolarization constant & 0.1 & $(1),(2)$ \\
$E$ & ON baseline activity level & 0.0 & $(1)$ \\
$E$ & OFF baseline activity level & 0.0 & $(1)$ \\
Stages 2 and 3: Simple and Complex Cells & & \\
A Scaling factor & 1000.0 & $(23)$ \\
$B \quad$ Complex cell threshold & 0.0 & $(23)$ \\
Stage $6:$ Bipole Cells, Long-Range Cooperation & & \\
$A_{0} \quad$ Output threshold & 0.08 & $(35)$ \\
$A_{1} \quad$ Output threshold & 0.07 & $(35)$ \\
$A_{2} \quad$ Output threshold & 0.05 & $(35)$ \\
Stage 9: Filling-in & & \\
$\epsilon$ & Permeability denominator factor & 2000.0 & $(39)-(42)$ \\
Stage 10: Combination of Scales & & \\
$N$ & ON double-opponent contrast coefficient \\
$P$ & OFF double-opponent contrast coefficient & 0.1 & $(54)$ \\
$M$ & Activation decay & 2.0 & $(54)$ \\
& & 20.0 & $(54)$
\end{tabular}

image, with parameters $U$ and $L$ factored out. If $\bar{E}>E$, then the second term in (8) increases as a function of a low-pass filtered and nonlinearly compressed transformation of image luminance, as in Method II of Grossberg and Wyse (1991). If $E=\bar{E}$, then this luminance-dependent term vanishes. In the case $E=\bar{E}=0$, Neumann (1993) proposed that the contrast term be combined with a luminance term

$$
l_{i j}^{g}=X_{i j}^{g}+\bar{X}_{i j}^{g}=\frac{(U-L) \sum_{(p, q)}\left(C_{i j p q}^{g}+S_{i j p q}^{g}\right) I_{p q}}{D+\sum_{(p, q)}\left(C_{i j p q}^{g}+S_{i j p q}^{g}\right) I_{p q}} .
$$

If the difference $d_{i j}^{g}$ in (8) were simply added to the sum $l_{i j}^{g}$ in (10), then the result would be $2 X_{i j}^{g}$, which reduces to model I of Grossberg and Todorović (1988). When $U>l$, model I generates responses similar to those of models II and III for the SAR imagery studies herein (see Figure 5). In Neumann (1993) and Pessoa, Mingolla, and Neumann (1995), the difference and sum terms are not merely added. Rather they are combined using a shunting equation

$$
\frac{d}{d t} b_{i j}^{g}=-M b_{i j}^{g}+l_{i j}^{g}+N S_{i j}^{g}-P \bar{S}_{i j}^{g},
$$

where $S_{i j}^{g}$ and $\bar{S}_{i j}^{g}$ are filled-in representations of the rectified variables $d_{i j}^{g}$ and $\bar{d}_{i j}^{g}$, respectively. See 
equations (49) and (50) below. At equilibrium,

$$
b_{i j}^{g}=\frac{l_{i j}^{g}+N S_{i j}^{g}}{M+P \bar{S}_{i j}^{g}} .
$$

In (12), ON and OFF double opponent contrasts modulate the baseline luminance in an upward and downward direction, respectively.

Figures 7 and 8 illustrate the result of using both ON and OFF channels in models II and III, respectively, on a 200x200 pixel example image (taken from the 400x400 bridge-overpass image shown in Figure 1a), at small, medium, and large scales from left to right. The top row shows the rectified ON channel response (5), the middle row the rectified OFF channel response (6), and the bottom row the net ON-minus-OFF double opponent response (8).

\subsection{Stage 2: Simple Model Cells}

The oriented simple cells model the first stage of oriented filtering in visual cortex. They use both the $\mathrm{ON}$ and $\mathrm{OFF}$ channels to gauge oriented contrast differences at each image location. An edge

elicits a strong response in the ON channel to one side and a strong OFF channel response on the other side. The simple cell filters are not just edge detectors, however. While they do produce an amplified response to abrupt edges, they are also capable of responding to relatively shallow image gradients. Simple cell outputs at scale $g$, position $(i, j)$, and orientation $k$ are modeled by the equations

$$
\begin{aligned}
& s_{i j k}^{R g}=\left[\left(R_{i j k}^{g}+\bar{L}_{i j k}^{g}\right)-\left(\bar{R}_{i j k}^{g}+L_{i j k}^{g}\right)\right]^{+}, \\
& s_{i j k}^{L g}=\left[-\left(R_{i j k}^{g}+\bar{L}_{i j k}^{g}\right)+\left(\bar{R}_{i j k}^{g}+L_{i j k}^{g}\right)\right]^{+},
\end{aligned}
$$

where

$$
\begin{aligned}
R_{i j k}^{g} & =\sum_{(p, q)} G_{\left(p, q+\frac{\sigma_{v g}}{2}, k\right)}^{g} X_{p q}^{g}, \\
\bar{R}_{i j k}^{g} & =\sum_{(p, q)} G_{\left(p, q+\frac{\sigma_{v g}}{2}, k\right)}^{g} \bar{X}_{p q}^{g}, \\
L_{i j k}^{g} & =\sum_{(p, q)} G_{\left(p, q-\frac{\sigma_{v g}}{2}, k\right)}^{g} X_{p q}^{g},
\end{aligned}
$$



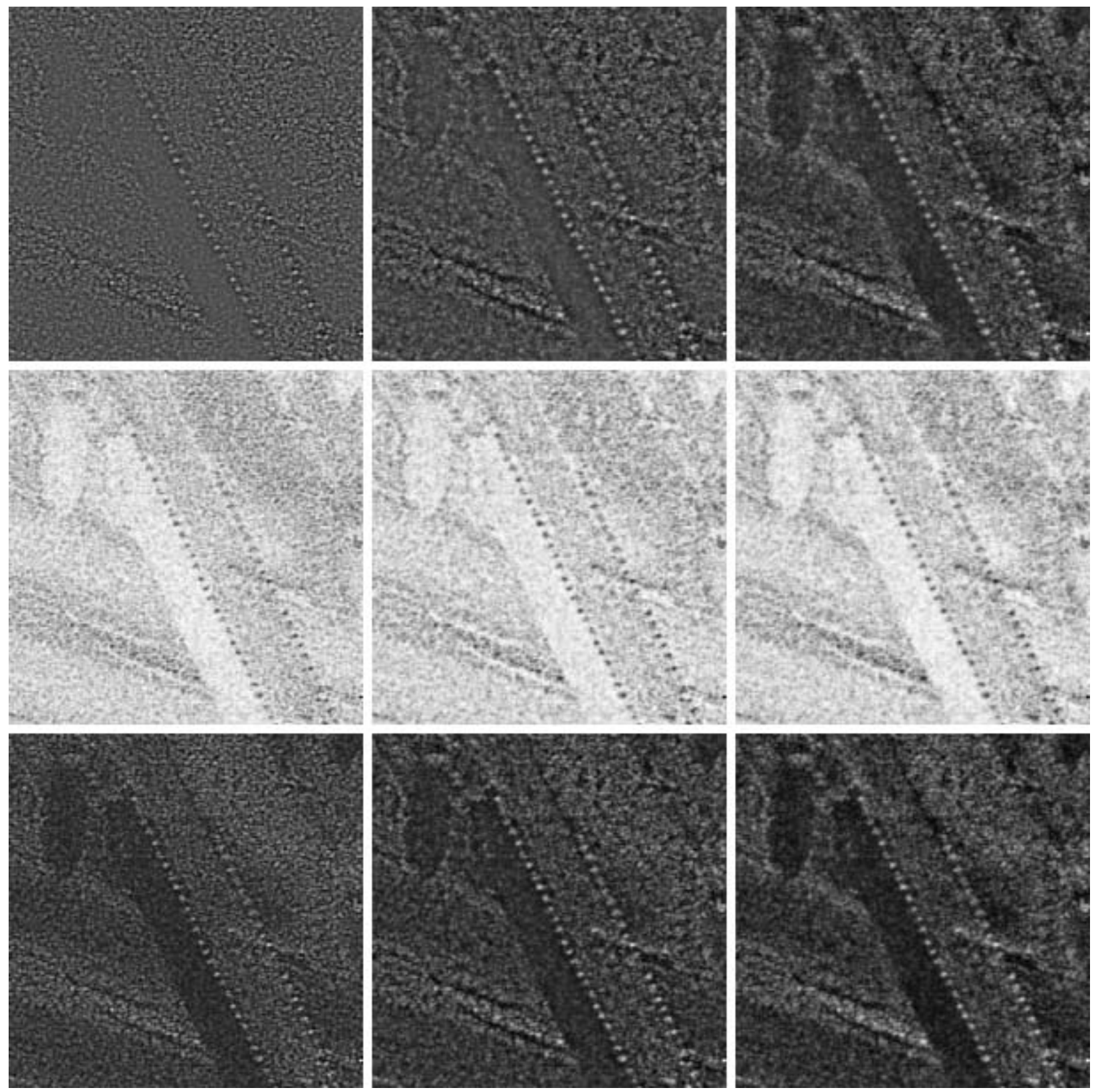

Figure 7: In Model II: (a) Rectified ON cell responses at small, medium, and large scales from left to right. (b) Rectified OFF cell responses. (c) Double-opponent ON-minus-OFF responses. 

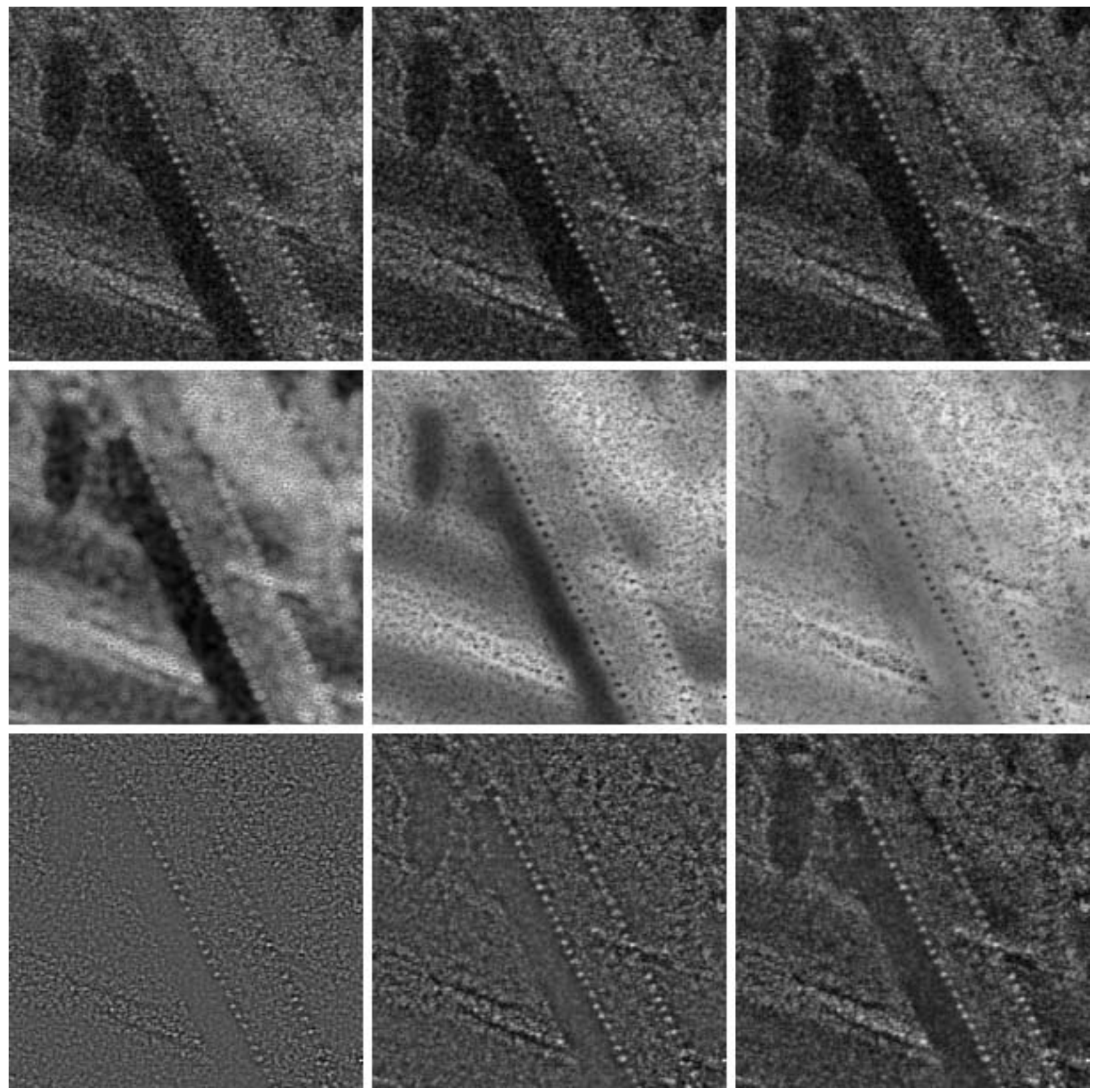

Figure 8: In Model III: (a) Rectified ON cell responses at small, medium, and large scales from left to right. (b) Rectified OFF cell responses. (c) Double-opponent ON-minus-OFF responses. 


$$
\bar{L}_{i j k}^{g}=\sum_{(p, q)} G_{\left(p, q-\frac{\sigma_{v g}}{2}, k\right)}^{g} \bar{X}_{p q}^{g},
$$

and

$$
G_{(p, q, k)}^{g}=\frac{1}{2 \pi \sigma_{h g} \sigma_{v g}} \exp \left\{-\frac{1}{2}\left(\left(\frac{p \cos \left(\frac{\pi k}{12}\right)-q \sin \left(\frac{\pi k}{12}\right)}{\sigma_{h g}}\right)^{2}+\left(\frac{p \sin \left(\frac{\pi k}{12}\right)+q \cos \left(\frac{\pi k}{12}\right)}{\sigma_{v g}}\right)^{2}\right)\right\}
$$

Equations (13) and (14) describe pairs of Stage 2 simple cells (see Figure 3) that are sensitive to opposite directions-of-contrast. Figure 9a shows the filters corresponding to the three spatial scales of a horizontally oriented $(k=0)$ simple cell $s_{i j k}^{L g}$ in $(14)$. Open circles denote where ON cells are weighted more strongly than OFF cells, and black circles the reverse, with circle area corresponding to the magnitude of the weighting difference between ON and OFF cells. Each simple cell thus receives excitatory input from an oriented array of ON cells and a spatially displaced but likeoriented array of OFF cells (Cruthirds et al.; 1992; Ferster, 1988; Liu et al., 1992; Miller, 1992). Pairs of simple cells that are sensitive to opposite contrast polarity, or direction-of-contrast, then compete (Figure 6b) to generate the net output signals in (13) and (14). This competition removes baseline activity differences in $\mathrm{ON}$ and $\mathrm{OFF}$ cells and weighs the relative advantage of opposite polarity simple cells. In the limiting case where there is no image contrast, there is no output from these cells.

The use of both ON and OFF cells to form boundaries overcomes complementary deficiencies of each detector in responding to changing contour curvatures and to dark or light noise pixels (Carpenter, Grossberg and Mehanian, 1989; Grossberg and Wyse, 1991). The net output signals in (13) and (14) include input from both ON and OFF cells within each oriented filter lobe, thus maximizing simple cell sensitivity to a given direction-of-contrast across the full range of ON and OFF cell activations. Stage 2 parameters consist of the standard deviations of the oriented filter defined in (19), which are $\sigma_{v g}$ and $\sigma_{h g}=3 \sigma_{v g}$ for $g=0,1,2$. See Table 1.

\subsection{Stage 3: Complex Cells}

A complex cell at scale $g$, position $(i, j)$, and orientation $k$ pools oriented contrast for both contrast polarities, or directions-of-contrast. Pooling is accomplished by summing the half-wave rectified outputs of simple cells at the same position and orientation but with opposite direction-of-contrast sensitivities:

$$
c_{i j k}^{g}=s_{i j k}^{L g}+s_{i j k}^{R g} .
$$



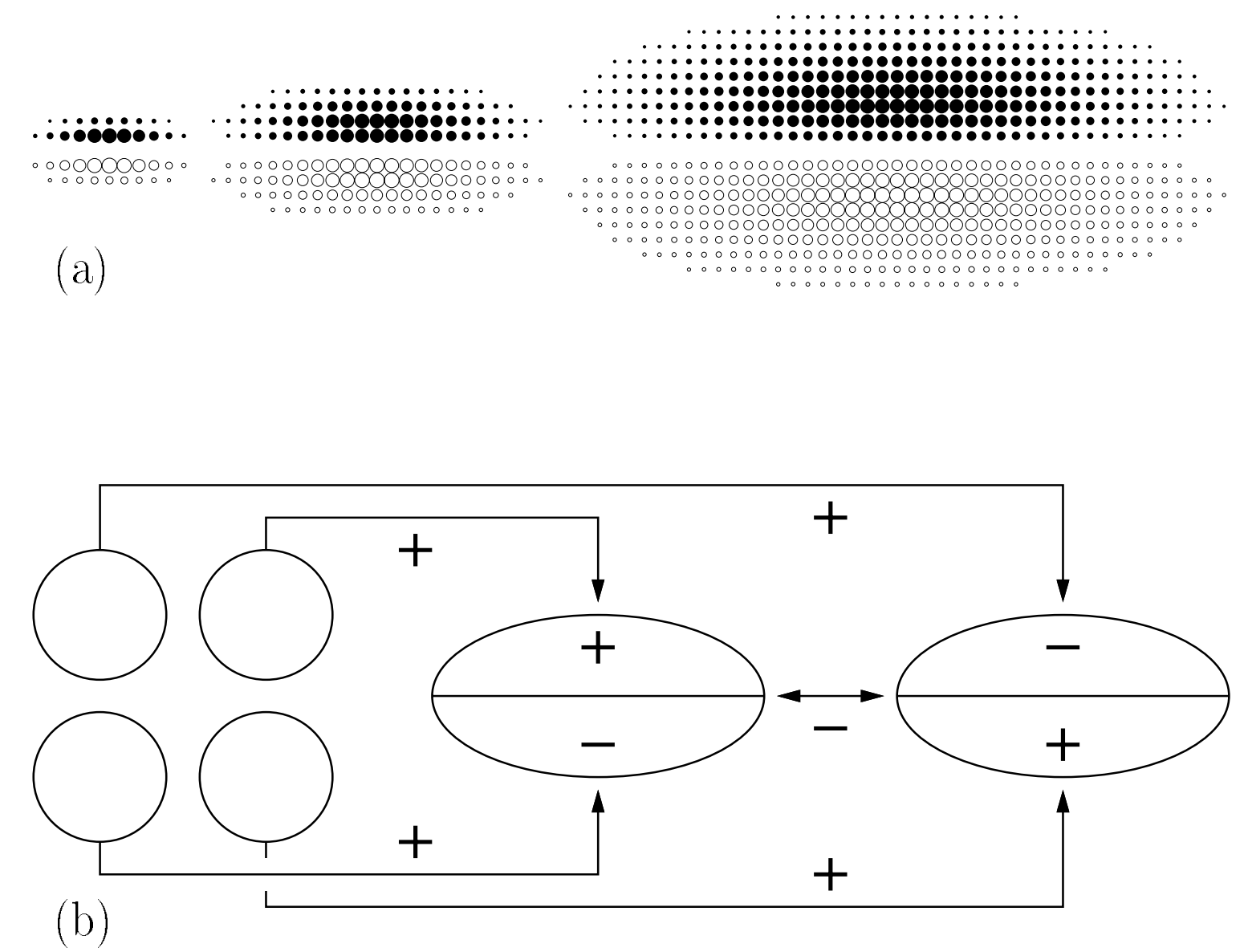

Figure 9: (a): Horizontal orientational filters used for Stage 2 simple cells at three spatial scales. Open circles denote where ON cells are weighted more strongly than OFF cells, and black circles the reverse, with circle area corresponding to the magnitude of the weighting difference between ON and OFF cells. (b): Circuitry for ON and OFF cell input to oriented simple cells of opposite direction-of-contrast. 
Equation (20) can be expressed directly in terms of Stage 1 ON and OFF cell outputs as

$$
c_{i j k}^{g}=\left|\sum_{(p, q)}\left(G_{\left(p, q+\frac{\sigma_{v g}}{2}, k\right)}^{g}-G_{\left(p, q-\frac{\sigma_{v g}}{2}, k\right)}^{g}\right)\left(X_{p q k}^{g}-\bar{X}_{p q k}^{g}\right)\right| \text {. }
$$

The top row of Figures 10 and 11 show the result of Stage 3 complex cell processing at small, medium, and large scales in response to models II and III, respectively. Here, image intensity represents the total activity summed across orientation of the complex cells at each position.

\subsection{Cooperative-Competitive Loop}

The complex cell output is passed into the Cooperative-Competitive Loop. This nonlinear feedback network detects, regularizes, and completes boundaries while suppressing image noise. The algorithm that implements the CC Loop iteratively applies six sequential processing stages to strengthen and complete consistent (i.e., colinear or near-colinear) boundary contours while deforming, sharpening, and thinning them (i.e., reducing variance across neighboring positions and orientations). The output of the six processing steps (Figure 3 ) are fed back into the first processing step to complete a single iteration of the loop. Five iterations of the $\mathrm{CC}$ Loop were used because functionally effective boundary completion could thereby be accomplished for the image resolution used.

The CC Loop was run independently at the three spatial scales. The Stage 5 hypercomplex output following five CC Loop iterations is shown in row two of Figures 10 and 11. Here, intensity represents the total amplitude $\sum_{k} y_{i j k}^{g}$ of cell activity at each position. Compare the complex cell activities in row 1 of Figure 10 or 11 with the hypercomplex cell activities after CC Loop feedback in row 2. The boundaries in row 2 are obviously sharper and more complete. The CC Loop is realized by the following processing stages.

\subsubsection{Stage 4: Hypercomplex Cells, Competition I (On-Center Off-Surround Inter- action Across Position)}

Output from Stage 3 as well as feedback from Stage 8 of the CC Loop are input into the equilibrium form of the following differential equation, in which cells of the same orientation at nearby positions 

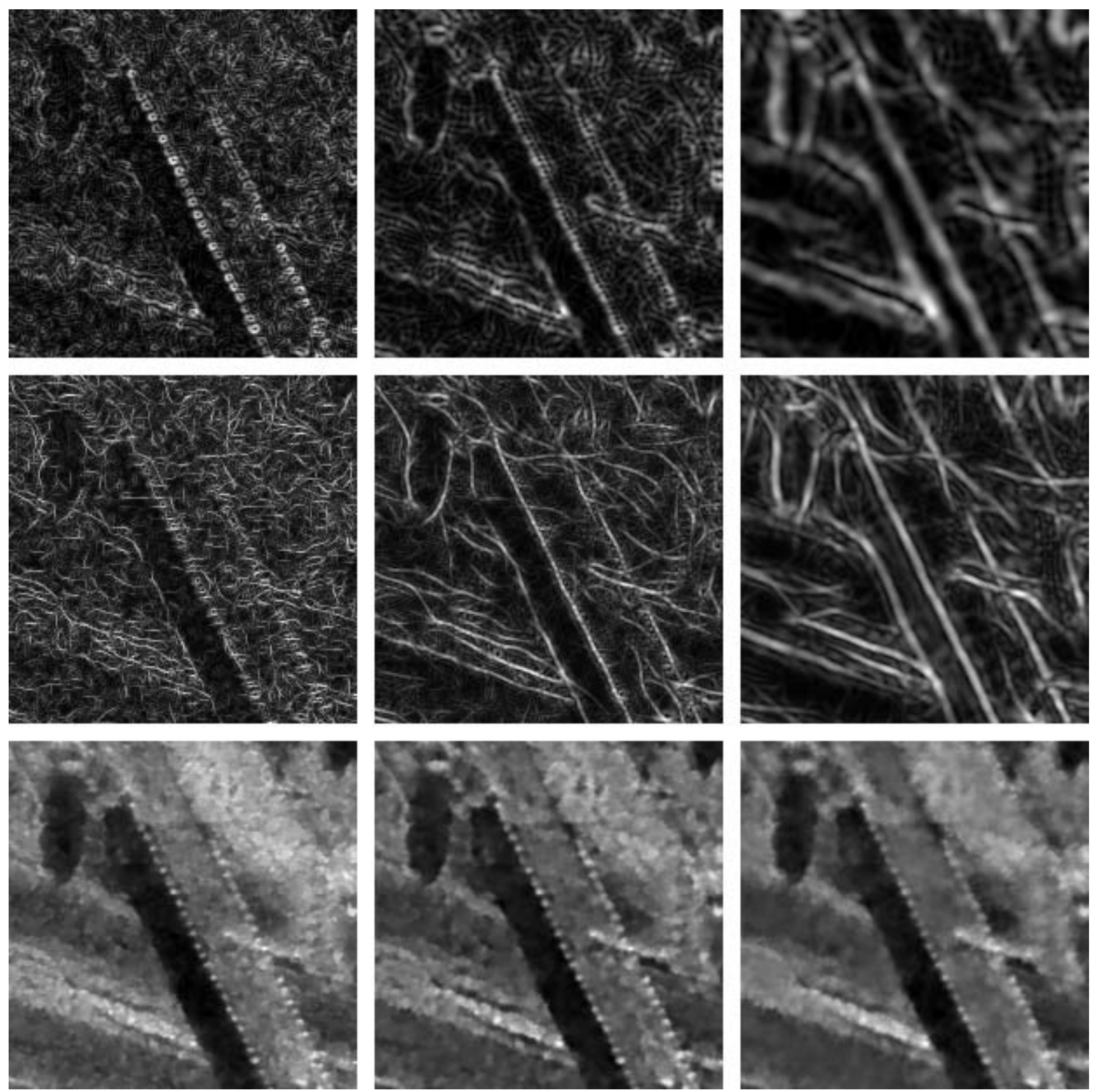

Figure 10: Top Row: Stage 3 complex cell processing at three scales of Model II. Intensity of each pixel depicts the total activity of the oriented complex cells at that position. Middle Row: Stage 5 hypercomplex cell processing at three spatial scales. Intensity of each pixel depicts the total activity of the cells at that position. Bottom Row: Stage 9 processing result at three different scales on example image. A linear combination of these images is used to obtain the final multiple-scale output in Figure 1d. 

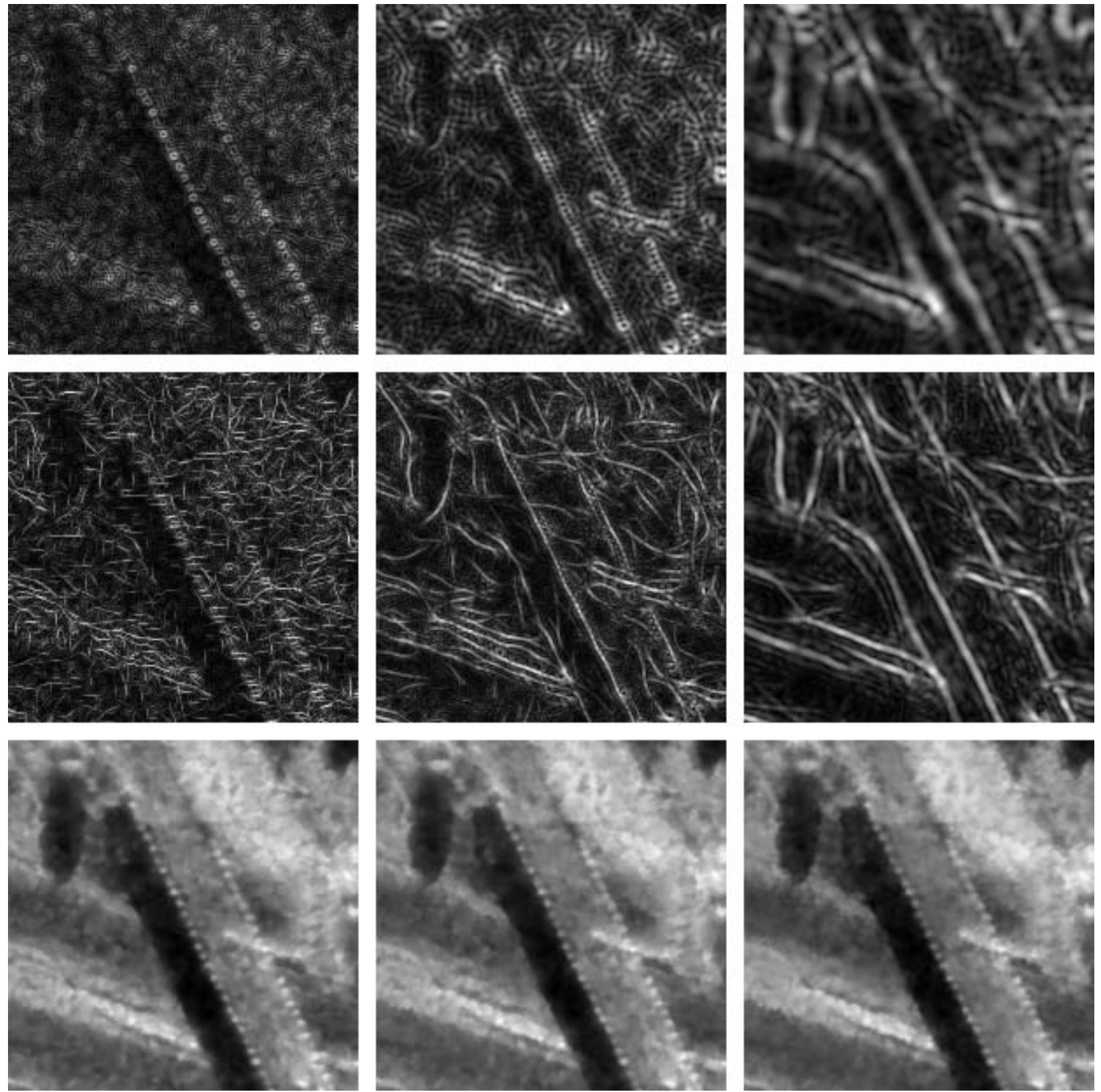

Figure 11: Same as Figure 10 for Model III. 
compete to help select the positionally best localized boundary:

$$
\frac{d}{d t} w_{i j k}^{g}=-D w_{i j k}^{g}+\sum_{(p, q)}\left[\left(U-w_{i j k}^{g}\right) C_{i j p q}^{g}-\left(w_{i j k}^{g}+L\right) S_{i j p q}^{g}\right] f\left(c_{p q k}^{g}\right)+T+h\left(v_{i j k}^{g}, g\right),
$$

where $C_{i j p q}^{g}$ and $S_{i j p q}^{g}$ are the center and surround Gaussian filters defined by (3) and (4),

$$
f(\omega)=A[\omega-B]^{+} \text {and } h(\omega, g)=E_{g} \omega
$$

and $v_{i j k}^{g}$ is defined in (37) below. The rectified equilibrium output signal generated by (22) is

$$
W_{i j k}^{g}=\frac{\left[\sum_{(p, q)}\left(U C_{i j p q}^{g}-L S_{i j p q}^{g}\right) f\left(c_{p q k}^{g}\right)+T+h\left(v_{i j k}^{g}\right)\right]^{+}}{D+\sum_{(p, q)}\left(C_{i j p q}^{g}+S_{i j p q}^{g}\right) f\left(c_{p q k}^{g}\right)}
$$

The feedforward spatial competition in the brackets of equation (23) realizes the endstopping operation that converts model complex cells into model hypercomplex cells. Parameter values for the three models are found in Tables 1-3.

\subsubsection{Stage 5: Hypercomplex Cells, Competition II (On-Center Off-Surround Inter- action Across Orientation)}

The second competitive stage of hypercomplex cells occurs across different orientations at the same position to select the most favored boundary orientations. Here,

$$
\frac{d}{d t} y_{i j k}=-D y_{i j k}^{g}+\sum_{r}\left[\left(U-y_{i j k}^{g}\right) C_{k r}-\left(y_{i j k}^{g}+L\right) S_{k r}\right] W_{i j r}^{g}
$$

where

$$
C_{k r}=\frac{C}{\sqrt{2 \pi \sigma_{c}^{2}}} \exp \left\{-\frac{(k-r)^{2}}{2 \sigma_{c}^{2}}\right\}
$$

and

$$
S_{k r}=\frac{S}{\sqrt{2 \pi \sigma_{s}^{2}}} \exp \left\{-\frac{(k-r)^{2}}{2 \sigma_{s}^{2}}\right\} .
$$

The equilibrium form of $(25)$ is

$$
y_{i j k}^{g}=\frac{\sum_{r}\left(U C_{k r}-L S_{k r}\right) W_{i j r}^{g}}{D+\sum_{r}\left(C_{k r}+S_{k r}\right) W_{i j r}^{g}} .
$$

See Tables 1-3 for parameter values. 
The positions and orientations selected by the feedforward competitive interactions among hypercomplex cells bias the cooperative grouping interactions among bipole cells that occur at Stage 6. Feedback from the bipole cells can, in turn, modify the orientations and positions selected by the feedforward competitive interactions via term $h\left(v_{i j k}^{g}\right)$ in (25). Thus, positions and orientations that receive only modest support directly from Stage 4 filters can win the competition at their position if they receive stronger net positive feedback from the CC Loop.

\subsubsection{Stage 6: Bipole Cells, Long-Range Cooperation (Statistical And-Gates)}

The cooperative grouping of the CC Loop is performed at Stage 6 by bipole cells that act like longrange statistical AND gates. In order for a horizontally oriented cooperative bipole cell to fire, both the left and right receptive fields of the cell need to receive input signals from the hypercomplex cells of Stage 5. When a bipole cell fires, it sends a top-down signal through Stages 7 and 8 to the hypercomplex cells of Stage 4, where it is combined with bottom-up information. This type of boundary completion can occur simultaneously across all orientations at all positions.

The oriented cooperation stage uses the "bow-tie" shaped bipole filters to achieve nonlinear cooperation between spatially separated cells having colinear or near-colinear orientations. The filters are sensitive to a range of orientations which increases with distance from the filter center. The amplitude of filter response also decreases with distance from the center, as well as with deviation from colinearity. Sufficient input must reach both lobes of the bipole cell for it to respond above threshold, thereby completing boundaries inwardly from pairs, or greater numbers, of input inducers. The oriented cooperation is accomplished via the differential equation

$$
\frac{d}{d t} z_{i j k}^{g}=-z_{i j k}^{g}+h\left(A_{i j k}^{g}\right)+h\left(B_{i j k}^{g}\right),
$$

which is implemented in the equilibrium form:

$$
z_{i j k}^{g}=h\left(A_{i j k}^{g}\right)+h\left(B_{i j k}^{g}\right),
$$

where

$$
h(\omega)=\frac{[\omega]^{+}}{K+[\omega]^{+}},
$$


(a)

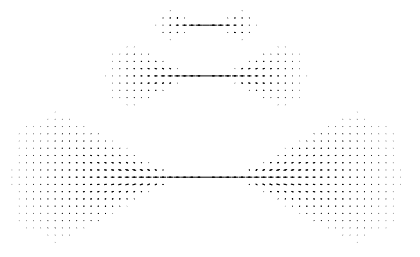

(b)

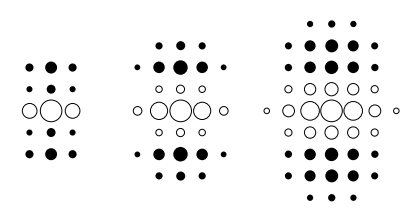

Figure 12: (a): Horizontal bipole filters at three spatial scales. The length of each oriented line segment is proportional to the filter coefficient at that location and orientation. (b): Horizontal feedback intra-orientational spatial sharpening filters at three spatial scales.

and (using the notation $\hat{r}$ for the orientation perpendicular to $r$ ),

$$
\begin{aligned}
A_{i j k}^{g}= & \sum_{(p, q, r)}\left(\left[y_{p q r}\right]^{+}-\left[y_{p q \hat{r}}\right]^{+}\right)\left[Z_{\left(2 p / C_{g}, 2 q / C_{g}, r, k\right)}\right]^{+}, \\
B_{i j k}^{g}= & \sum_{(p, q, r)}\left(\left[y_{p q r}\right]^{+}-\left[y_{p q \hat{r}}\right]^{+}\right)\left[-Z_{\left(2 p / C_{g}, 2 q / C_{g}, r, k\right)}\right]^{+}, \\
Z_{(p, q, r, k)}= & \operatorname{sgn}\{p\} \exp \left\{-\beta\left(p^{2}+q^{2}\right)\right\} \exp \left\{-\mu\left(\frac{q}{p^{2}}\right)^{2}\right\} . \\
& \cos ^{\gamma}\left\{\frac{(k-r) \pi}{K}-\operatorname{sgn}\{p\} \arctan \left(\frac{2 q}{p}, p\right)\right\} .
\end{aligned}
$$

Equation (34) is composed of three parts which determine how the bipole filter values decrease as a function of $(1)$ the distance from the center of the filter: $\exp \left\{-\beta\left(p^{2}+q^{2}\right)\right\} ;$ (2) spatial deviation from colinearity: $\exp \left\{-\mu\left(\frac{q}{p^{2}}\right)^{2}\right\}$; and (3) orientational deviation from colinearity: $\cos ^{\gamma}\left\{\frac{(k-r) \pi}{K}-\operatorname{sgn}\{p\} \arctan \left(\frac{2 q}{p}, p\right)\right\}$. The CC Loop is run independently at three different scales, with bipole filters defined by (34) sampled at sizes of $C_{g}, g=0,1,2$, given in the Tables. Figure 12 shows these three bipole scales at the horizontal orientation $(k=0)$. In Figure 13a, each line orientation represents filter orientation, and line length represents filter magnitude at the corresponding position. 
The output of each cooperative bipole cell is given by

$$
f\left(z_{i j k}^{g}\right)=\left[z_{i j k}^{g}-A_{g}\right]^{+} .
$$

The bipole cell outputs are next orientationally and spatially sharpened in order to compensate for the orientational and spatial fuzziness of the bipole cells receptive fields.

\subsubsection{Stage 7: Hypercomplex/Bipole Cells, Feedback Competition II (On-Center Off-Surround Interaction Across Orientation)}

The cooperative bipole cells compete across orientation at each position to select the cooperatively favored orientations. Specifically, Stage 6 output is passed to the equation

$$
u_{i j k}^{g}=\frac{\sum_{r}\left(U C_{k r}-L S_{k r}\right) f\left(z_{i j r}^{g}\right)}{D+\sum_{r}\left(C_{k r}+S_{k r}\right) f\left(z_{i j r}^{g}\right)},
$$

where all parameter values are the same as in Stage 5 .

\subsubsection{Stage 8: Hypercomplex/Bipole Cells, Feedback Competition I (On-Center Off- Surround Interaction Across Position)}

A final competitive feedback stage is used to achieve spatial sharpening while selecting the most favored spatial positions among all the nearby cooperative cells that are tuned for the same orientation. This is accomplished by convolving each oriented output from Stage 7 with an anisotropic DOG filter, elongated in the preferred orientation. Specifically,

$$
v_{i j k}^{g}=\sum_{(p, q)} u_{p q k}^{g} F_{i j p q k}^{g},
$$

where $F$ is an oriented filter made up of the difference of a center and two flanking Gaussians,

$$
F_{i j p q k}^{g}=G_{(i-p, j-q, k)}^{g}-\frac{1}{2}\left(G_{\left(i-p, j-q+\sigma_{v}, k\right)}^{g}+G_{\left(i-p, j-q-\sigma_{v}, k\right)}^{g}\right)
$$

where $G_{(p, q, k)}^{g}$ is the Gaussian kernel defined in (19). See Tables 1-3 for parameter values. Figure

8 b shows horizontal $F$ filters at the three spatial scales. The output $v_{i j k}^{g}$ from the final CC Loop stage feeds back to the first CC Loop stage, as in (24). The bottom-up and top-down CC Loop signals hereby resonate to choose the statistically most favored boundary segmentation. 


\subsection{Stage 9: Filling-In}

The BCS produces boundary signals that act as barriers to diffusion within the FCS. As in Grossberg and Mingolla (1985b), BCS output signals are derived from Stage 5 of the CC Loop. Those boundary signals act to gate diffusion of signals in the filling-in domains of Stage 9 that are activated by $\mathrm{ON}$ and OFF cell output of Stage 1. For image pixels through which no boundary signals pass, resulting intensity values become more homogeneous as the diffusion evolves. Where boundary signals intervene, however, they inhibit the diffusion, leaving a resulting activity difference on either side of the boundary signal. These boundary signals are organized into a form-sensitive mesh that is called a boundary web (Grossberg, 1987a; Grossberg and Mingolla, 1987). Boundary webs can track the statistics of edges, textures, and shading. This is how boundary-gated filling-in achieves its sensitivity to the form of each unique configuration of image inputs. After ON and OFF filling-in occurs, the outputs are combined as in Figure 3b-d to generate the net surface representation of that scale.

Inputs from Stage 1 undergo a nonlinear diffusion process at State 9 within compartments defined by boundary signals. In particular, boundary signals create high resistance barriers to filling-in. The diffusion equations in response to individual ON and OFF cell outputs are (Cohen and Grossberg, 1984; Grossberg and Todorović, 1988):

\section{ON Filling-In}

$$
\frac{d}{d t} s_{i j}^{g}=-D s_{i j}^{g}+\sum_{p, q \in N_{i j}}\left(s_{p q}^{g}-s_{i j}^{g}\right) P_{p q i j}^{g}+X_{i j}^{g},
$$

where $X_{i j}^{g}$ is defined by $(5)$.

\section{OFF Filling-In}

$$
\frac{d}{d t} \bar{s}_{i j}^{g}=-D \bar{s}_{i j}^{g}+\sum_{p, q \in N_{i j}}\left(\bar{s}_{p q}^{g}-\bar{s}_{i j}^{g}\right) P_{p q i j}^{g}+\bar{X}_{i j}^{g},
$$

where $\bar{X}_{i j}^{g}$ is defined by (6). At equilibrium,

$$
s_{i j}^{g}=\frac{X_{i j}^{g}+\sum_{p, q \in N_{i j}} s_{p q}^{g} P_{p q i j}^{g}}{D+\sum_{p, q \in N_{i j}} P_{p q i j}^{g}},
$$


and

$$
\bar{s}_{i j}^{g}=\frac{\bar{X}_{i j}^{g}+\sum_{p, q \in N_{i j}} \bar{s}_{p q}^{g} P_{p q i j}^{g}}{D+\sum_{p, q \in N_{i j}} P_{p q i j}^{g}} .
$$

The boundary-gated permeabilities obey

$$
P_{p q i j}^{g}=\frac{\delta}{1+\epsilon\left(y_{p q}^{g}+y_{i j}^{g}\right)},
$$

where

$$
y_{i j}^{g}=\sum_{k} y_{i j k}^{g}
$$

Note that the permeability $P_{p q i j}^{g}$ decreases as the boundary signals $y_{p q}^{g}$ and $y_{i j}^{g}$ from Stage 5 increase. In other words, the diffusion gate closes as the boundary gets strong. The nearest-neighbor sources and sinks of diffusion in (40)-(41) are:

$$
N_{i j}=\{(i, j-1),(i-1, j),(i+1, j),(i, j+1)\} .
$$

In model I, the filled-in ON activities $s_{i j}^{g}$ are used to form the outputs. In model II, the filled-in OFF activities $\bar{s}_{i j}^{g}$ are subtracted from the filled-in ON activities $s_{i j}^{g}$ to derive the outputs. Alternatively, the net double-opponent response $X_{i j}^{g}-\bar{X}_{i j}^{g}$ in (8) could be used to fill-in a single diffusion network. In model III, all the terms $d_{i j}^{g}$ in (8), $\bar{d}_{i j}^{g}$ in (9), and $l_{i j}^{g}$ in (10) are used to fill-in net $\mathrm{ON}, \mathrm{OFF}$, and luminance responses;

$$
\begin{gathered}
\frac{d}{d t} S_{i j}^{g}=-D S_{i j}^{g}+\sum_{p, q \in N_{i j}}\left(S_{p q}^{g}-S_{i j}^{g}\right) P_{p q i j}^{g}+\left[d_{i j}^{g}\right]^{+}, \\
\frac{d}{d t} \bar{S}_{i j}^{g}=-D \bar{S}_{i j}^{g}+\sum_{p, q \in N_{i j}}\left(\bar{S}_{p q}^{g}-\bar{S}_{i j}^{g}\right) P_{p q i j}^{g}+\left[\bar{d}_{i j}^{g}\right]^{+}, \\
\frac{d}{d t} L_{i j}^{g}=-D L_{i j}^{g}+\sum_{p, q \in N_{i j}}\left(L_{p q}^{g}-L_{i j}^{g}\right) P_{p q i j}^{g}+l_{i j}^{g},
\end{gathered}
$$


which are simulated in equilibrium form,

$$
\begin{gathered}
S_{i j}^{g}=\frac{\left[d_{i j}^{g}\right]^{+} \sum_{p, q \in N_{i j}} S_{p q}^{g} P_{p q i j}^{g}}{D+\sum_{p, q \in N_{i j}} P_{p q i j}^{g}} \\
\bar{S}_{i j}^{g}=\frac{\left[\bar{d}_{i j}^{g}\right]^{+}+\sum_{p, q \in N_{i j}} \bar{S}_{p q}^{g} P_{p q i j}^{g}}{D+\sum_{p, q \in N_{i j}} P_{p q i j}^{g}} \\
L_{i j}^{g}=\frac{\left[l_{i j}^{g}\right]^{+}+\sum_{p, q \in N_{i j}} L_{p q}^{g} P_{p q i j}^{g}}{D+\sum_{p, q \in N_{i j}} P_{p q i j}^{g}}
\end{gathered}
$$

Equations (41) and (42) are implemented for 400 iterations. The filling-in parameter values are $D=0.05, \delta=10.0, \epsilon=1000.0, n=400$. The net outputs for the three scales $g=1,2,3$, are shown for models II and III in row 3 of Figures 10 and 11. Note that, although the medium and large scale BCS boundaries in row 2 of Figure 10 and 11 cannot distinguish the small posts on the bridge, these posts are recovered in all the FCS filled-in surface representations, including the medium and large scale images. This is true due to two properties operating together: (1) A narrow on-center is used to discount the illuminant across all scales, and thus to distinguish the posts across all scales at the ON and OFF cell outputs depicted in Figure 5. (2) The medium and large scale boundaries "cover" the post locations, and thus trap their local contrasts within their boundary web. See Grossberg and Mingolla (1987) and Grossberg and Todorović (1988) for related uses of boundary web properties.

\subsection{Stage 10: Combination of Scales}

The final output image is attained by a weighted combination of filled-in double-opponent surface representations at different scales. Weighting coefficients are selected so that the variances of the three filled-in double-opponent component images are approximately equal. The multiple-scale output surface is thus computed as

\section{Model I:}

$$
O_{i j}=s_{i j}^{0}+s_{i j}^{1}+s_{i j}^{2}
$$




\section{Model II:}

$$
O_{i j}=5\left(s_{i j}^{0}-\bar{s}_{i j}^{0}\right)+4\left(s_{i j}^{1}-\bar{s}_{i j}^{1}\right)+2\left(s_{i j}^{2}-\bar{s}_{i j}^{2}\right) .
$$

\section{Model III:}

$$
O_{i j}=\sum_{g=0}^{2} \frac{L_{i j}^{g}+N S_{i j}^{g}}{M+P \bar{S}_{i j}^{g}}
$$

The quality of the final output image is not sensitive to the exact values of the respective weighting coefficients. We chose values such that each scale has an approximately equal contribution "by eye" to producing the final combined-scale output. A more sophisticated multiple-scale interaction which has been proposed to achieve figure-ground separation lays the foundation for future research (Grossberg, 1994).

\section{Comparison with prior methods}

The BCS/FCS results were compared with previously published methods for speckle noise reduction. To facilitate comparison, the unprocessed SAR values $I$ were passed through the compressive function

$$
f(I)=\frac{I}{D+I}
$$

where $D=2,000$, in order to produce imagery of roughly the same grey-level distributions as the BCS/FCS Stage 1 output, before processing by the alternative noise reduction methods. The alternative methods considered are: smoothing with a median filter (Scollar, Weidner, and Huang, 1984), adaptive averaging with a sigma filter (Lee, 1983), and smoothing with a geometric filter (Crimmins, 1985). The parameters of these methods are set to obtain a similar net amount of smoothing - as determined by informal observation - as the BCS/FCS, in order to evaluate how well they remove noise while retaining actual image features. Because it tends to suppress outliers, the median filter is a sensible method for reducing speckle noise (Scollar, et al., 1984). A 3x3 median filter was applied for 3 iterations. Alternatively, averaging with a mean filter blurs real edges too much. This problem is addressed with the sigma filter, which only averages those pixels with intensity within two standard deviations of the center pixel. However, this approach leaves many outliers, which are due to speckle noise, untouched. This problem is addressed by locally averaging 

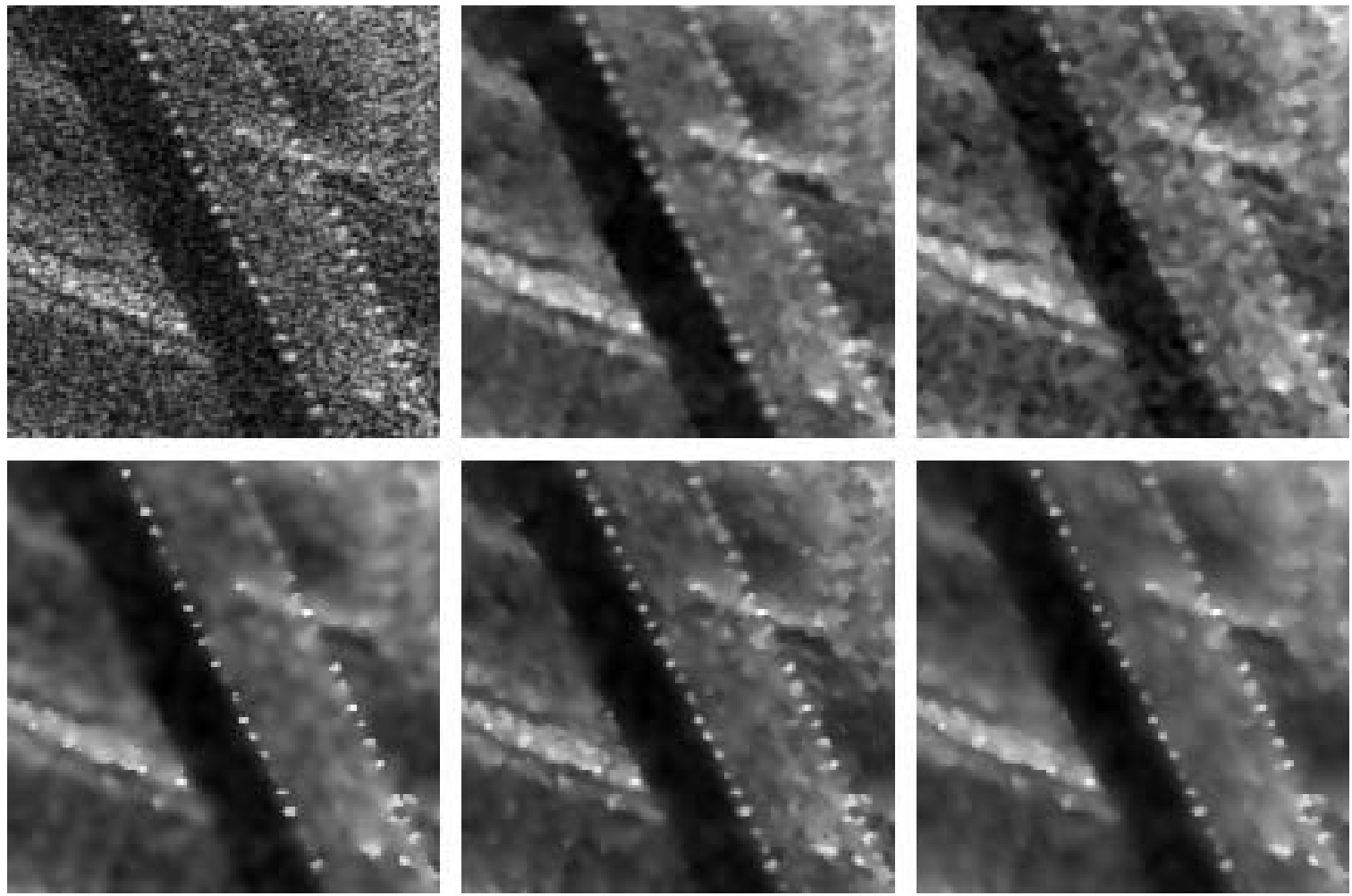

Figure 13: Comparison of speckle noise reduction methods. (a) Top Left: SAR image (compressed for viewing) of scene with overpass over New York State Thruway, taken from image in Figure 1. (b) Top Middle: Multi-scale BCS/FCS. (c) Top Right: 3 iterations of 3x3 median filter. (c) Bottom Left: Adaptive averaging using 2 iterations of 5x5 sigma filter. (d) Bottom Middle: 3 iterations of geometric filter. (e) Bottom Right: 4 iterations of geometric filter. 

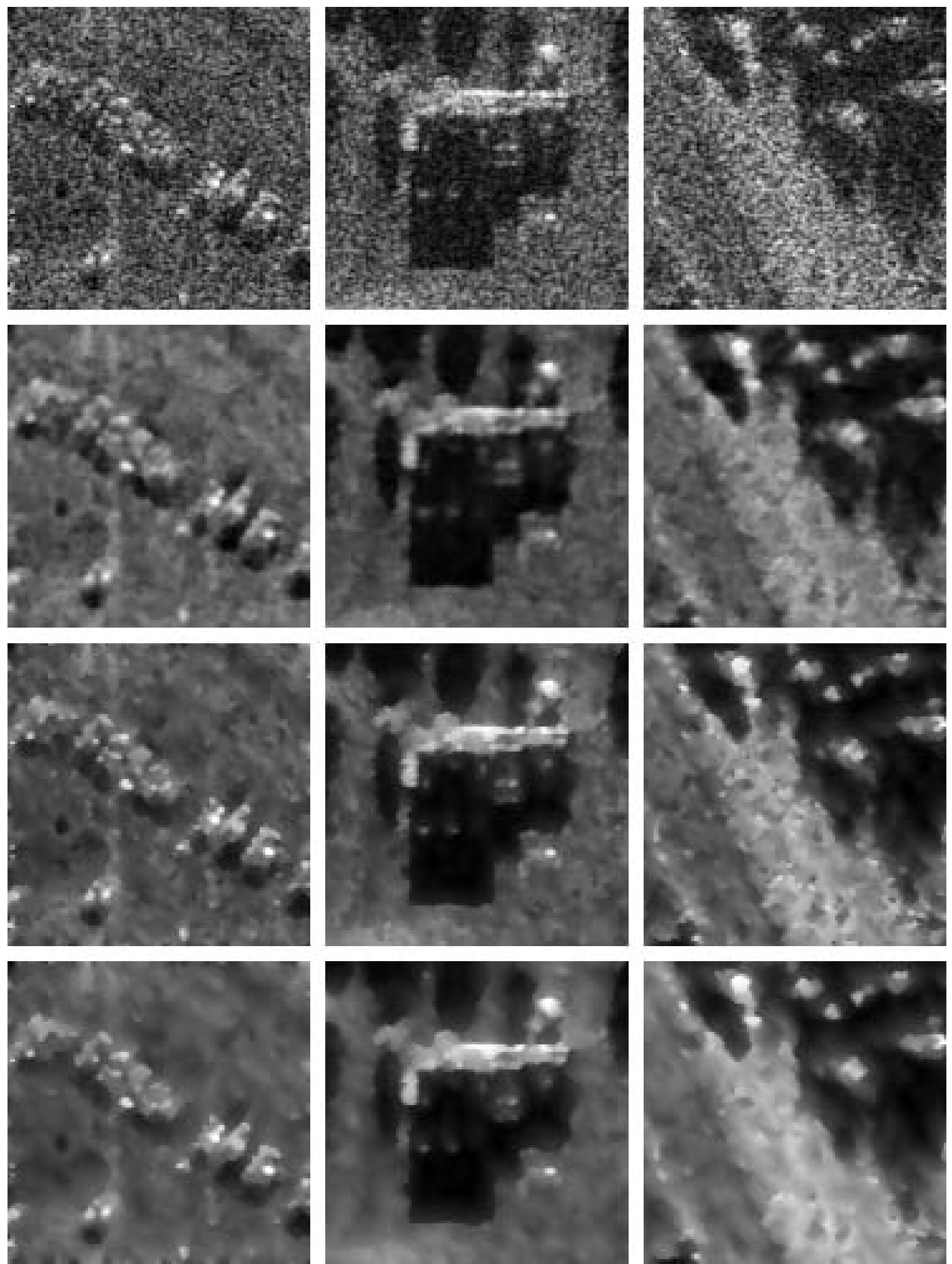

Figure 14: Comparison of BCS/FCS and geometric filter. Top Left: SAR image (compressed for viewing) of cars lined up in parking lot; Top Middle: a house with shadow; Top Right: a mixture of trees and shadows, with grass below and a road on the left. Second row: Corresponding BCS/FCS results. Third row: Corresponding geometric filter results, with 3 iterations. Fourth row: Corresponding geometric filter results, with 4 iterations. 

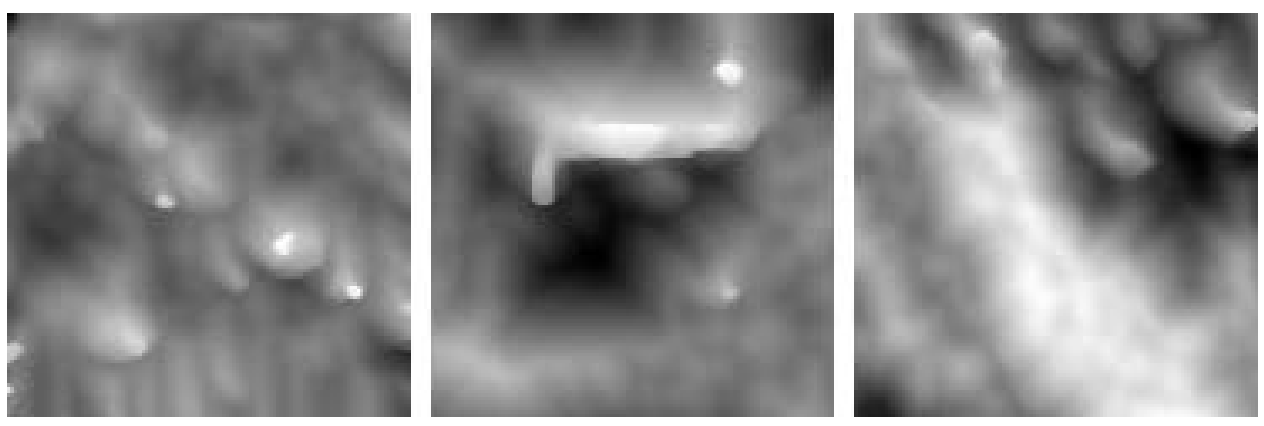

Figure 15: Geometric filter results, with 10 iterations, corresponding to Figure 14. 
those pixels for which $K$ or fewer other pixels in the averaging window lie within two standard deviations (Lee, 1983). Adaptive averaging is done for 2 iterations, using a $5 \times 5$ sigma filter, with the standard deviation estimated at a relatively flat image region, and a threshold of $K=3$ for removing spot noise (see Lee, 1983). Another method for speckle noise reduction, the geometric filter, iteratively enforces a minimum constraint for curvature, in pixel intensity space, between neighboring pixels (Crimmins, 1985). Each iteration of the geometric filter involves two successive applications of four nearest-neighbor intensity curvature rules, in four directions 45 degrees apart, horizontally, diagonally, vertically, and diagonally. The first application reduces the curvature from above, filling in holes or narrow valleys. The second application reduces curvature from below, reducing spikes or narrow ridges.

Figure 13 (top left) shows a section of the image from Figure 1 following compression of signal values by (55), used as input to the noise reduction methods. This image contains an overpass of the New York State Thruway. Note that the detail of the overpass guardrails is maintained by the BCS/FCS (top middle), while regions that are homogeneous with the exception of speckle noise are smoothed over. The median filter method (top right) and adaptive averaging method (bottom left) do not do as well at maintaining important detail while smoothing away noise. The geometric filter, after 3 iterations (bottom center), and 4 iterations (bottom right), also does a good job at smoothing noise while maintaining detail.

Because the BCS/FCS and geometric filtering methods do the best at speckle noise reduction on the image in Figure 13, they alone were evaluated on additional images. Figure 14 (top left) shows a SAR image of cars lined up in a parking lot, Figure 14 (top middle) a house with shadow, and Figure 14 (top right) a mixture of trees and shadows, with grass below and a road on the left. The second row of Figure 14 shows the corresponding BCS/FCS results, the third row the geometric filter results with 3 iterations, and the four row the geometric filter results with 4 iterations.

Comparing the three systems, the BCS/FCS arguably produces results that are smoother while being more true to the actual imagery. An important consideration in evaluating the alternative approaches is that the BCS/FCS reliably produces results like those shown in Figure 14, whereas the geometric filter iteratively smooths the image. Therefore, when using a geometric filter, the user must choose how many iterations to apply to achieve the desired level of smoothness for each set of images. Crimmins (1985) reported that 10 iterations of the geometric filter seem to be optimal for the imagery of that study. However, when applied to the imagery of Figure 14, 10 iterations produces extremely washed-out looking results, as shown in Figure 15. Thus the BCS provides a 
more robust method for generating boundary and surface representations that do not degrade as the number of iterations increases. 


\section{References}

\section{References}

Arrington, K.F. (1994). The temporal dynamics of brightness filling-in. Vision Research, 34, $3371-3387$.

Carpenter, G., Grossberg, S., and Mehanian, C. (1989). Invariant recognition of cluttered scenes by a self-organizing ART architecture: CORT-X boundary segmentation. Neural Networks, 2 , $169-181$.

Cohen, M. and Grossberg, S. (1984). Neural dynamics of brightness perception: Features, boundaries, diffusion, and resonance. Perception and Psychophysics, 36, 428-456.

Crimmins, T. (1985). Geometric filter for speckle reduction. Applied Optics, 24, 1438-1443.

Cruthirds, D., Gove, A., Grossberg, S., Mingolla, E., Nowak, N., and Williamson, J. (1992). Processing of synthetic aperture radar images by the Boundary Contour System and Feature Contour System. Proceedings of the International Joint Conference on Neural Networks (IJCNN-92), IV-414-419.

Ferster, D. (1988). Spatially opponent excitation and inhibition in simple cells of the cat visual cortex. Journal of Neuroscience, 8, 1172-1180.

Grossberg, S. (1973). Contour enhancement, short term memory, and constancies in reverberating neural networks. Studies in Applied Mathematics, 52, 217-257.

Grossberg, S. (1984). Outline of a theory of brightness, color, and form perception. In E. Degreef and J. van Buggenhaut (Eds.), Trends in Mathematical Psychology. Amsterdam: North Holland. 
Grossberg, S. (1987a). Cortical dynamics of three-dimensional form, color, and brightness perception, I: Monocular theory. Perception and Psychophysics, 41, 87-116.

Grossberg, S. (1987b). Cortical dynamics of three-dimensional form, color, and brightness perception, II: Binocular theory. Perception and Psychophysics, 41, 117-158.

Grossberg, S. (1994). 3-D vision and figure-ground separation by visual cortex. Perception and Psychophysics, 55, 48-120.

Grossberg, S. and Mingolla, E. (1985a). Neural dynamics of form perception: Boundary completion, illusory figures, and neon color spreading. Psychological Review, 92, 173-211.

Grossberg, S. and Mingolla, E. (1985b). Neural dynamics of perceptual grouping: Textures, boundaries, and emergent segmentations. Perception and Psychophysics, 38, 141-171.

Grossberg, S. and Mingolla, E. (1987). Neural dynamics of surface perception: Boundary webs, illuminants, and shape-from-shading. Computer Vision, Graphics, and Image Processing, 37, $116-165$.

Grossberg, S., Mingolla, E. and Todorović, D. (1989). A neural network architecture for preattentive vision. IEEE Transactions on Biomedical Engineering, 36, 65-84.

Grossberg, S. and Todorović, D. (1988). Neural dynamics of 1-D and 2-D brightness perception: A unified model of classical and recent phenomena. Perception and Psychophysics, 43, 241-277.

Grossberg, S. and Wyse, L. (1991). A neural network architecture for figure-ground separation of connected scenic figures. Neural Networks, 4, 723-742. 
Lee, J. (1983). A simple speckle smoothing algorithm for synthetic aperture radar images. IEEE Transactions on Systems, Man, and Cybernetics, SMC-13, 85-89.

Liu, Z., Gaska, J.P., Jacobson, L.D., and Pollen, D.A. (1992). Interneuronal interaction between members of quadrature phase and anti-phase pairs in the cat's visual cortex. Vision Research, 32, 1193-1198.

Miller, K.D. (1992). Development of orientation columns via competition between ON- and OFFcenter inputs. NeuroReport, 3, 73-76.

Mollon, J.D. and Sharpe, L.T. (1983). Color Vision. New York, NY: Academic Press.

Munsen, D. Jr., O’Brien, J., and Jenkins, W. (1983). A tomographic formulation of spotlight-mode synthetic aperture radar. Proceedings of the IEEE, 71(8), 917-925.

Munsen, D. Jr., and Visentin, R. L. (1989). A signal processing view of strip-mapping synthetic aperture radar. IEEE Transactions on Acoustics, Speech, and Signal Processing, 37(12), 21312147.

Neumann, H. (1993). Toward a computational architecture for unified visual contrast and brightness perception: I. Theory and model. In Proceedings of the World Conference on Neural Networks (WCNN-93), I, 87-91. Hillsdale, NJ: Erlbaum.

Novak, L., Burl, M., Chaney, R., and Owirka, G. (1990). Optimal processing of polarimetric synthetic-aperture radar imagery. The Lincoln Laboratory Journal, 3(2), 273-290.

Paradiso, M.A. and Nakayama, K. (1991). Brightness perception and filling-in. Vision Research, $31,1221-1236$.

Pessoa, L., Mingolla, E., and Neumann, H. (1995). A contrast- and luminance-driven multiscale network model of brightness perception. Vision Research, in press. 
Scollar, L., Weidner, B., and Huang, T. (1984). Image enhancement using the median and the interquartile distance. Computer Vision, Graphics, and Image Processing, 25, 236-251.

von der Heydt, R., Peterhans, E., and Baumgartner, G. (1984). Illusory contours and cortical neuron responses. Science, 224, 1260-1262. 